ANALYSIS \& PDE

Volume $2 \quad$ No. $3 \quad 2009$

TRISTAN ROY

GLOBAL EXISTENCE OF SMOOTH SOLUTIONS OF A 3D LOG-LOG ENERGY-SUPERCRITICAL WAVE EQUATION 


\title{
GLOBAL EXISTENCE OF SMOOTH SOLUTIONS OF A 3D LOG-LOG ENERGY-SUPERCRITICAL WAVE EQUATION
}

\author{
TRISTAN ROY
}

We prove global existence of smooth solutions of the 3D log-log energy-supercritical wave equation

$$
\partial_{t t} u-\triangle u=-u^{5} \log ^{c}\left(\log \left(10+u^{2}\right)\right)
$$

with $0<c<8 / 225$ and smooth initial data $\left(u(0)=u_{0}, \partial_{t} u(0)=u_{1}\right)$. First we control the $L_{t}^{4} L_{x}^{12}$ norm of the solution on an arbitrary size time interval by an expression depending on the energy and an a priori upper bound of its $L_{t}^{\infty} \tilde{H}^{2}\left(\mathbb{R}^{3}\right)$ norm, with $\tilde{H}^{2}\left(\mathbb{R}^{3}\right):=\dot{H}^{2}\left(\mathbb{R}^{3}\right) \cap \dot{H}^{1}\left(\mathbb{R}^{3}\right)$. The proof of this long time estimate relies upon the use of some potential decay estimates and a modification of an argument by Tao. Then we find an a posteriori upper bound of the $L_{t}^{\infty} \tilde{H}^{2}\left(\mathbb{R}^{3}\right)$ norm of the solution by combining the long time estimate with an induction on time of the Strichartz estimates.

\section{Introduction}

We shall consider the defocusing log-log energy-supercritical wave equation

$$
\partial_{t t} u-\Delta u=-f(u)
$$

where $u: \mathbb{R} \times \mathbb{R}^{3} \rightarrow \mathbb{R}$ is a real-valued scalar field and $f(u):=u^{5} g(u)$ with $g(u):=\log ^{c}\left(\log \left(10+u^{2}\right)\right)$, $0<c<8 / 225$. Classical solutions of (1-1) are solutions that are infinitely differentiable and compactly supported in space for each fixed time $t$. It is not difficult to see that classical solutions of (1-1) satisfy the energy conservation law

$$
E:=\frac{1}{2} \int_{\mathbb{R}^{3}}\left(\partial_{t} u(t, x)\right)^{2} d x+\frac{1}{2} \int_{\mathbb{R}^{3}}|\nabla u(t, x)|^{2} d x+\int_{\mathbb{R}^{3}} F(u(t, x)) d x
$$

where $F(u):=\int_{0}^{u} f(v) d v$. Classical solutions of (1-1) enjoy three symmetry properties that we use throughout this paper:

- time translation invariance: if $u$ is a solution of (1-1) and $t_{0}$ is a fixed time then $\tilde{u}(t, x):=u\left(t-t_{0}, x\right)$ is also a solution of (1-1);

- space translation invariance: if $u$ is a solution of (1-1) and $x_{0}$ is a fixed point lying in $\mathbb{R}^{3}$ then $\tilde{u}(t, x):=u\left(t, x-x_{0}\right)$ is also a solution of $(1-1)$;

- time reversal invariance: if $u$ is a solution to (1-1) then $\tilde{u}(t, x):=u(-t, x)$ is also a solution.

MSC2000: 35Q55.

Keywords: global regularity, log-log energy supercritical wave equation. 
The defocusing log-log energy-supercritical wave equation (1-1) is closely related to the power-type defocusing wave equations, namely,

$$
\partial_{t t} u-\Delta u=-|u|^{p-1} u .
$$

Solutions of (1-3) have an invariant scaling

$$
u(t, x) \rightarrow u^{\lambda}(t, x):=\frac{1}{\lambda^{2 /(p-1)}} u\left(\frac{t}{\lambda}, \frac{x}{\lambda}\right)
$$

and (1-3) is $s_{c}$-critical, where $s_{c}:=\frac{3}{2}-\frac{2}{p-1}$. Thus the $\dot{H}^{s_{c}}\left(\mathbb{R}^{3}\right) \times \dot{H}^{s_{c}-1}\left(\mathbb{R}^{3}\right)$ norm of $\left(u(0), \partial_{t} u(0)\right)$ is invariant under scaling, i.e.,

$$
\begin{aligned}
\left\|u^{\lambda}(0)\right\|_{\dot{H}^{s_{c}\left(\mathbb{R}^{3}\right)}} & =\|u(0)\|_{\dot{H}^{s_{c}\left(\mathbb{R}^{3}\right)}}, \\
\left\|\partial_{t} u^{\lambda}(0)\right\|_{\dot{H}^{s_{c}-1}\left(\mathbb{R}^{3}\right)} & =\left\|\partial_{t} u(0)\right\|_{\dot{H}^{s_{c}-1}\left(\mathbb{R}^{3}\right)}
\end{aligned}
$$

If $p=5$, then $s_{c}=1$ and this is why the quintic defocusing cubic wave equation

$$
\partial_{t t} u-\triangle u=-u^{5}
$$

is called the energy-critical equation. If $1<p<5$ then $s_{c}<1$ and (1-3) is energy-subcritical while if $p>5$ then $s_{c}>1$ and (1-3) is energy-supercritical. Notice that for every $p>5$ there exists two positive constant $\lambda_{1}(p), \lambda_{2}(p)$ such that

$$
\lambda_{1}(p)|u|^{5} \leq|f(u)| \leq \lambda_{2}(p) \max \left(1,|u|^{p}\right) .
$$

This is why (1-1) is said to belong to the group of barely supercritical equations. There is another way to see that. Notice that a simple integration by part shows that

$$
F(u) \sim \frac{u^{6}}{6} g(u),
$$

and consequently the nonlinear potential term of the energy $\int_{\mathbb{R}^{3}} F(u) d x \sim \int_{\mathbb{R}^{3}} u^{6} g(u) d x$ just barely fails to be controlled by the linear component, in contrast to (1-5).

The energy-critical wave equation (1-5) has received a great deal of attention. Grillakis [1990; 1992] established global existence of smooth solutions (global regularity) of this equation with smooth initial data $u(0)=u_{0}, \partial_{t} u(0)=u_{1}$. His work followed that of Rauch [1981, part I] for small data and that of Struwe [1988] on the spherically symmetric case. Later Shatah and Struwe [1993] gave a simplified proof of this result. Kapitanski [1994] and, independently, Shatah and Struwe [1994] proved global existence of solutions with data $\left(u_{0}, u_{1}\right)$ in the energy class.

We are interested in proving global regularity of (1-1) with smooth initial data $\left(u_{0}, u_{1}\right)$. By standard persistence of regularity results it suffices to prove global existence of solutions

$$
u \in \mathscr{C}\left([0, T], \tilde{H}^{2}\left(\mathbb{R}^{3}\right)\right) \cap \mathscr{C}^{1}\left([0, T], H^{1}\left(\mathbb{R}^{3}\right)\right),
$$

with data $\left(u_{0}, u_{1}\right) \in \tilde{H}^{2}\left(\mathbb{R}^{3}\right) \times H^{1}\left(\mathbb{R}^{3}\right)$. Here the following space

$$
\tilde{H}^{2}\left(\mathbb{R}^{3}\right):=\dot{H}^{2}\left(\mathbb{R}^{3}\right) \cap \dot{H}^{1}\left(\mathbb{R}^{3}\right) .
$$


In view of the local well-posedness theory [Lindblad and Sogge 1995], standard limit arguments and the finite speed of propagation it suffices to find an a priori upper bound of the form

$$
\left\|\left(u(T), \partial_{t} u(T)\right)\right\|_{\tilde{H}^{2}\left(\mathbb{R}^{3}\right) \times H^{1}\left(\mathbb{R}^{3}\right)} \leq C_{1}\left(\left\|u_{0}\right\|_{\tilde{H}^{2}\left(\mathbb{R}^{3}\right)},\left\|u_{1}\right\|_{H^{1}\left(\mathbb{R}^{3}\right)}, T\right)
$$

for all times $T>0$ and for classical solutions $u$ of (1-1) with smooth and compactly supported data $\left(u_{0}, u_{1}\right)$. Here $C_{1}$ is a constant depending only on $\left\|u_{0}\right\|_{\tilde{H}^{2}\left(\mathbb{R}^{3}\right)},\left\|u_{1}\right\|_{H^{1}\left(\mathbb{R}^{3}\right)}$ and the time $T$.

The global behavior of the solutions of the supercritical wave equations is poorly understood, mostly because of the lack of conservation laws in $\tilde{H}^{2}\left(\mathbb{R}^{3}\right)$. Nevertheless Tao [2007] was able to prove global regularity for another barely supercritical equation, namely

$$
\partial_{t t} u-\Delta u=-u^{5} \log \left(2+u^{2}\right)
$$

with radial data. The main result of this paper is:

Theorem 1. The solution of (1-1) with smooth data $\left(u_{0}, u_{1}\right)$ exists for all time. Moreover there exists a nonnegative constant $M_{0}=M_{0}\left(\left\|u_{0}\right\|_{\tilde{H}^{2}\left(\mathbb{R}^{3}\right)},\left\|u_{1}\right\|_{H^{1}\left(\mathbb{R}^{3}\right)}\right)$ depending only on $\left\|u_{0}\right\|_{\tilde{H}^{2}\left(\mathbb{R}^{3}\right)}$ and $\left\|u_{1}\right\|_{H^{1}\left(\mathbb{R}^{3}\right)}$ such that

$$
\|u\|_{L_{t}^{\infty} \tilde{H}^{2}\left(\mathbb{R} \times \mathbb{R}^{3}\right)}+\left\|\partial_{t} u\right\|_{L_{t}^{\infty} H^{1}\left(\mathbb{R} \times \mathbb{R}^{3}\right)} \leq M_{0} .
$$

We recall some basic properties and estimates. Let $Q$ be a function, let $J$ be an interval and let $t_{0} \in J$ be a fixed time. If $u$ is a classical solution of the more general problem $\partial_{t t} u-\Delta u=Q$ then $u$ satisfies the Duhamel formula

$$
u(t)=u_{l, t_{0}}(t)+u_{n l, t_{0}}(t), \quad t \in J,
$$

with $u_{l, t_{0}}, u_{n l, t_{0}}$ denoting the linear part and the nonlinear part respectively of the solution starting from $t_{0}$. Recall that

$$
u_{l, t_{0}}(t)=\cos \left(t-t_{0}\right) D u\left(t_{0}\right)+\frac{\sin \left(t-t_{0}\right) D}{D} \partial_{t} u\left(t_{0}\right)
$$

and

$$
u_{n l, t_{0}}(t)=-\int_{t_{0}}^{t} \frac{\sin \left(t-t^{\prime}\right) D}{D} Q\left(t^{\prime}\right) d t^{\prime},
$$

with $D$ the multiplier defined by $\widehat{D f}(\xi):=|\xi| \widehat{f}(\xi)$. An explicit formula for $\left(\left(\sin \left(t-t^{\prime}\right) D\right) / D\right) Q\left(t^{\prime}\right)$ and $t \neq t^{\prime}$ is

$$
\left[\frac{\sin \left(t-t^{\prime}\right) D}{D} Q\left(t^{\prime}\right)\right](x)=\frac{1}{4 \pi\left|t-t^{\prime}\right|} \int_{\left|x-x^{\prime}\right|=\left|t-t^{\prime}\right|} Q\left(t^{\prime}, x^{\prime}\right) d S\left(x^{\prime}\right) .
$$

For a proof see [Sogge 1995]. We recall that $u_{l, t_{0}}$ satisfies

$$
\partial_{t t} u_{l, t_{0}}-\Delta u_{l, t_{0}}=0, \quad u_{l, t_{0}}\left(t_{0}\right)=u\left(t_{0}\right), \quad \partial_{t} u_{l, t_{0}}\left(t_{0}\right)=\partial_{t} u\left(t_{0}\right),
$$

while $u_{n l, t_{0}}$ is the solution of

$$
\partial_{t t} u_{n l, t_{0}}-\Delta u_{n l, t_{0}}=Q, \quad u_{n l, t_{0}}\left(t_{0}\right)=0, \quad \partial_{t} u_{n l, t_{0}}\left(t_{0}\right)=0 .
$$

We recall the Strichartz estimate [Ginibre and Velo 1995; Keel and Tao 1998; Lindblad and Sogge 1995; Sogge 1995]

$$
\|u\|_{L_{t}^{q} L_{x}^{r}\left(J \times \mathbb{R}^{3}\right)} \lesssim\left\|\partial_{t} u\left(t_{0}\right)\right\|_{L_{x}^{2}\left(\mathbb{R}^{3}\right)}+\left\|\nabla u\left(t_{0}\right)\right\|_{L_{x}^{2}\left(\mathbb{R}^{3}\right)}+\|Q\|_{L_{t}^{1} L_{x}^{2}\left(J \times \mathbb{R}^{3}\right)}
$$


if $(q, r)$ is wave admissible, that is, $(q, r) \in(2, \infty] \times[2, \infty]$ and $1 / q+3 / r=1 / 2$.

We set some notation that appears throughout the paper. We write $C=C\left(a_{1}, \ldots, a_{n}\right)$ if $C$ only depends on the parameters $a_{1}, \ldots, a_{n}$. We write $A \lesssim B$ if there exists a universal nonnegative constant $C^{\prime}>0$ such that $A \leq C^{\prime} B . A=O(B)$ means $A \lesssim B$. More generally we write $A \lesssim a_{1}, \ldots, a_{n} B$ if there exists a nonnegative constant $C^{\prime}=C\left(a_{1}, \ldots, a_{n}\right)$ such that $A \leq C^{\prime} B$. We say that $C^{\prime \prime}$ is the constant determined by $\lesssim$ in $A \lesssim a_{1}, \ldots, a_{n} B$ if $C^{\prime \prime}$ is the smallest constant among the $C^{\prime}$ s such that $A \leq C^{\prime} B$. We write $A \ll_{a_{1}, \ldots, a_{n}} B$ if there exists a universal nonnegative small constant $c=c\left(a_{1}, \ldots, a_{n}\right)$ such that $A \leq c B$. Similar notions are defined for $A \gtrsim B, A \gtrsim a_{1}, \ldots, a_{n} B$ and $A \gg B$. In particular we say that $C^{\prime \prime}$ is the constant determined by $\gtrsim$ in $A \gtrsim B$ if $C^{\prime \prime}$ is the largest constant among the $C^{\prime}$ s such that $A \geq C^{\prime} B$. If $x$ is number then $x+$ and $x-$ are slight variations of $x: x+:=x+\alpha \epsilon$ and $x-:=x-\beta \epsilon$ for some $\alpha>0, \beta>0$ and $0<\epsilon \ll 1$.

Let $\Gamma_{+}$denote the forward light cone

$$
\Gamma_{+}=\{(t, x): t>|x|\},
$$

and if $J=[a, b]$ is an interval, let $\Gamma_{+}(J)$ denote the light cone truncated to $J$, that is,

$$
\Gamma_{+}(J):=\Gamma_{+} \cap\left(J \times \mathbb{R}^{3}\right) .
$$

Let $e(t)$ denote the local energy, that is,

$$
e(t):=\frac{1}{2} \int_{|x| \leq t}\left(\partial_{t} u(t, x)\right)^{2} d x+\frac{1}{2} \int_{|x| \leq t}|\nabla u(t, x)|^{2} d x+\int_{|x| \leq t} F(u(t, x)) d x .
$$

If $u$ is a solution of (1-1) then by using the finite speed of propagation and the Strichartz estimates we have

$$
\|u\|_{L_{t}^{q} L_{x}^{r}\left(\Gamma_{+}(J)\right)} \lesssim\|\nabla u(b)\|_{L_{x}^{2}\left(\mathbb{R}^{3}\right)}+\left\|\partial_{t} u(b)\right\|_{L_{x}^{2}\left(\mathbb{R}^{3}\right)}+\|Q\|_{L_{t}^{1} L_{x}^{2}\left(\Gamma_{+}(J)\right)}
$$

if $(q, r)$ is wave admissible. If $J_{1}:=\left[a_{1}, a_{2}\right]$ and $J_{2}:=\left[a_{2}, a_{3}\right]$ then we also have

$$
\|u\|_{L_{t}^{q} L_{x}^{r}\left(\Gamma_{+}\left(J_{1}\right)\right)} \lesssim\left\|\nabla u\left(a_{3}\right)\right\|_{L_{x}^{2}\left(\mathbb{R}^{3}\right)}+\left\|\partial_{t} u\left(a_{3}\right)\right\|_{L_{x}^{2}\left(\mathbb{R}^{3}\right)}+\|Q\|_{L_{t}^{1} L_{x}^{2}\left(\Gamma_{+}\left(J_{1} \cup J_{2}\right)\right)} .
$$

We recall also the well-known Sobolev embeddings. If $h$ is a smooth function then

$$
\|h\|_{L^{\infty}\left(\mathbb{R}^{3}\right)} \lesssim\|h\|_{\tilde{H}^{2}\left(\mathbb{R}^{3}\right)}
$$

and

$$
\|h\|_{L^{6}\left(\mathbb{R}^{3}\right)} \lesssim\|\nabla h\|_{L^{2}\left(\mathbb{R}^{3}\right)} .
$$

If $u$ is the solution of (1-1) with data $\left(u_{0}, u_{1}\right) \in \tilde{H}^{2}\left(\mathbb{R}^{3}\right) \times H^{1}\left(\mathbb{R}^{3}\right)$, then we get from (1-22)

$$
E \lesssim\left\|u_{0}\right\|_{\tilde{H}^{2}\left(\mathbb{R}^{3}\right)}^{2} \max \left(1,\left\|u_{0}\right\|_{\tilde{H}^{2}\left(\mathbb{R}^{3}\right)}^{4} g\left(\left\|u_{0}\right\|_{\tilde{H}^{2}\left(\mathbb{R}^{3}\right)}\right)\right) .
$$

We shall use the Paley-Littlewood technology. Let $\phi(\xi)$ be a bump function adapted to $\left\{\xi \in \mathbb{R}^{3}:|\xi| \leq 2\right\}$ and equal to one on $\left\{\xi \in \mathbb{R}^{3}:|\xi| \leq 1\right\}$. If $(M, N) \in 2^{\mathbb{Z}} \times 2^{\mathbb{Z}}$ are dyadic numbers then the Paley-Littlewood projection operators $P_{M}, P_{<N}$ and $P_{\geq N}$ are defined in the Fourier domain by

$$
\widehat{P_{M} f}(\xi):=\left(\phi\left(\frac{\xi}{M}\right)-\phi\left(\frac{\xi}{2 M}\right)\right) \hat{f}(\xi), \quad \widehat{P_{<N} f}(\xi):=\sum_{M<N} \widehat{P_{M} f}(\xi), \quad \widehat{P_{\geq N} f}(\xi):=\sum_{M \geq N} \widehat{P_{M} f}(\xi) .
$$

The inverse Sobolev inequality can be stated as follows: 
Proposition 2 (Inverse Sobolev inequality [Tao 2006]). Let $g$ be a smooth function such that

$$
\|g\|_{\dot{H}^{1}\left(\mathbb{R}^{3}\right)} \lesssim E^{1 / 2} l, \quad\left\|P_{\geq N} g\right\|_{L_{x}^{6}\left(\mathbb{R}^{3}\right)} \gtrsim \eta,
$$

for some real number $\eta>0$ and for some dyadic number $N>0$. Then there exists a ball $B(x, r) \subset \mathbb{R}^{3}$ with $r=O(1 / N)$ such that we have the mass concentration estimate

$$
\int_{B(x, r)}|g(y)|^{2} d y \gtrsim \eta^{3} E^{-1 / 2} r^{2} .
$$

We also recall a result that shows that the mass of solutions of (1-1) can be locally in time controlled. Proposition 3 (Local mass is locally stable [Tao 2006]). Let $J$ be a time interval, let $t, t^{\prime} \in J$ and let $B(x, r)$ be a ball. Let $u$ be a solution of (1-1). Then

$$
\left(\int_{B(x, r)}\left|u\left(t^{\prime}, y\right)\right|^{2} d y\right)^{1 / 2}=\left(\int_{B(x, r)}|u(t, y)|^{2} d y\right)^{1 / 2}+O\left(E^{1 / 2}\left|t-t^{\prime}\right|\right) .
$$

This result, proved for (1-5) in [Tao 2006], is also true for (1-1). Indeed the proof relied upon the fact that the $L^{2}\left(\mathbb{R}^{3}\right)$ norm of the velocity of the solution of (1-5) at time $t$ is bounded by the square root of its energy, which is also true for the solution of (1-1) (by (1-2) and (1-7)).

Now we make some comments with respect to Theorem 1. If the function $g$ were a positive constant, it would be easy to prove that the solution of (1-1) with data $\left(u_{0}, u_{1}\right)$ lies in $\tilde{H}^{2}\left(\mathbb{R}^{3}\right) \times H^{1}\left(\mathbb{R}^{3}\right)$, since we have a good global theory for (1-5). Therefore we can hope to prove global well-posedness for $g$ slowly increasing to infinity, by extending the technology to prove global well-posedness for (1-5). Notice also that Tao [2006] found that the solution $u$ of (1-5) satisfies

$$
\|u\|_{L_{t}^{4} L_{x}^{12}\left(\mathbb{R} \times \mathbb{R}^{3}\right)} \lesssim \tilde{E}^{\tilde{E}^{O(1)}},
$$

with $\tilde{E}$ the energy of $u$. The structure of $g$ is a double log: it is, roughly speaking, the inverse function of the towel exponential bound in (1-27).

Now we explain the main ideas of this paper.

Tao [2006] was able to bound on arbitrary long time intervals the $L_{t}^{4} L_{x}^{12}$ norm of solutions of the energy-critical equation (1-5) by a quantity that depends exponentially on their energy. This estimate can be viewed as a long time estimate. Unfortunately we cannot expect to prove a similar result for (1-1) since we are not in the energy-critical regime. However we shall prove the following proposition:

Proposition 4 (Long time estimate). Let $J=\left[t_{1}, t_{2}\right]$ be a time interval. Let $u$ be a classical solution of (1-1). Assume that

$$
\|u\|_{L_{t}^{\infty} \tilde{H}^{2}\left(J \times \mathbb{R}^{3}\right)} \leq M
$$

for some $M \geq 0$. Then there exist three constants $C_{L, 0}>0, C_{L, 1}>0$ and $C_{L, 2}>0$ such that

- if $E \ll \frac{1}{g^{1 / 2}(M)}$ (small energy regime) then

$$
\|u\|_{L_{t}^{4} L_{x}^{12}\left(J \times \mathbb{R}^{3}\right)}^{4} \leq C_{L, 0}
$$

- if $E \gtrsim \frac{1}{g^{1 / 2}(M)}$ (large energy regime) then

$$
\|u\|_{L_{t}^{4} L_{x}^{12}\left(J \times \mathbb{R}^{3}\right)}^{4} \leq\left(C_{L, 1}(E g(M))\right)^{C_{L, 2}\left(E^{193 / 4+} g^{225 / 8+}(M)\right)} .
$$


This proposition shows that we can control the $L_{t}^{4} L_{x}^{12}\left(J \times \mathbb{R}^{3}\right)$ norm of solutions of (1-1) by their energy and an a priori bound of their $L_{t}^{\infty} \tilde{H}^{2}\left(J \times \mathbb{R}^{3}\right)$ norm. We would like to control the pointwisein-time $\tilde{H}^{2}\left(\mathbb{R}^{3}\right) \times H^{1}\left(\mathbb{R}^{3}\right)$ norm of $u$ on an interval [0,T], with $T$ arbitrarily large. This is done by an induction on time. We assume that this norm is controlled on $[0, T]$ by a number $M_{0}$. Then by continuity we can find a slightly larger interval $\left[0, T^{\prime}\right]$ such that this norm is bounded by (say) $2 M_{0}$ on $\left[0, T^{\prime}\right]$. This is our a priori bound. We subdivide $\left[0, T^{\prime}\right]$ into subintervals where the $L_{t}^{4} L_{x}^{12}$ norm of $u$ is small and we control the pointwise-in-time $\tilde{H}^{2}\left(\mathbb{R}^{3}\right) \times H^{1}\left(\mathbb{R}^{3}\right)$ norm of $u$ on each of these subintervals (see Lemma 6). Since $g$ varies slowly we can estimate the number of intervals of this partition by using Proposition 4 and we can prove a posteriori that $\|u(t)\|_{\tilde{H}^{2}\left(\mathbb{R}^{3}\right)}+\left\|\partial_{t} u(t)\right\|_{\tilde{H}^{1}\left(\mathbb{R}^{3}\right)}$ is bounded on $\left[0, T^{\prime}\right]$ by $M_{0}$, provided that $M_{0}$ is large enough; see Section 2.

The proof of Proposition 4 is a modification of the argument used in [Tao 2006] to establish a towerexponential bound of the $L_{t}^{4} L_{x}^{12}\left(J \times \mathbb{R}^{3}\right)$ norm of $v$, the solution of (1-5). We divide $J$ into subintervals $J_{i}$ where the $L_{t}^{4} L_{x}^{12}$ norm of $u$, the solution of (1-1), is "substantial". Then by using the Strichartz estimates and the Sobolev embedding (1-22) we notice that the $L_{t}^{\infty} L_{x}^{6}\left(J_{i} \times \mathbb{R}^{3}\right)$ norm of $u$ is also substantial, more precisely, we find a lower bound that depends on the energy $E$ and $g(M)$. Then by Proposition 2 we can localize a bubble where the mass concentrates and we prove that the size of these subintervals is also substantially large. Tao [2006] used the mass concentration to construct a solution $\tilde{v}$ of (1-5) that has a smaller energy than $v$ and that coincides with $v$ outside a cone. The idea behind that is to use an induction on the levels of energy, due to Bourgain [1999], and the small energy theory following from the Strichartz estimates in order to control the $L_{t}^{4} L_{x}^{12}$ norm of $v$ outside a cone. Unfortunately it seems almost impossible to apply this procedure to our problem. Indeed the energy of the constructed solution $\tilde{u}$ is smaller than the energy $E$ of $u$ by an amount that depends on $E$ but also on $g(M)$ and therefore an induction on the levels of the energy is possible if the $L_{t}^{\infty} \tilde{H}^{2}\left(J \times \mathbb{R}^{3}\right)$ norm of $\tilde{u}$ can be controlled by $M$, which is far from being trivial. It turns out that we do not need to use the Bourgain induction method. Indeed since we know that the size of the subintervals $J_{i}$ s is substantially large and since we have a good control of the $L_{t}^{4} L_{x}^{12}$ norm on these subintervals it suffices to find an upper bound of the size of their union in order to conclude. To this end we divide a cone containing the ball where the mass concentrates and the $J_{i}$ s into truncated-in-time cones where the $L_{t}^{4} L_{x}^{12}$ norm of $u$ is substantial. Let $\tilde{J}_{1}, \tilde{J}_{2}, \ldots$ be the sequence of time intervals resulting from this partition. The mass concentration helps us to control the size of the first time interval $\tilde{J}_{1}$. By using an asymptotic stability result we can prove, roughly speaking, that if we consider two successive subintervals $\tilde{J}_{j}, \tilde{J}_{j+1}$ resulting from this partition of the cone then the size of $\tilde{J}_{j+1}$ can be controlled by the size of $\tilde{J}_{j}$; see (3-34). But a potential energy decay estimate shows that if the size of the union of the $J_{i} \mathrm{~s}$ is too large then we can find a large subinterval $\left[t_{1}^{\prime}, t_{2}^{\prime}\right]$ such that the $L_{t}^{4} L_{x}^{12}$ norm of $u$ on the cone truncated to $\left[t_{1}^{\prime}, t_{2}^{\prime}\right]$ is small. Therefore $\left[t_{1}^{\prime}, t_{2}^{\prime}\right]$ cannot be covered by many $\tilde{J}_{j}$ s and one of them is very large in comparison with its predecessor, which contradicts (3-34). At the end of the process we can find an upper bound of the size of the union of the subintervals $J_{i} \mathrm{~s}$ and consequently we can control the $L_{t}^{4} L_{x}^{12}$ norm of $u$ on the interval $J$.

Remark 5. We will frequently use the $x+$ and $x$ - notations. Indeed the point $(2, \infty)$ is not wave admissible. Therefore we will work with the point $(2+, \infty-)$ : see (5-6) and (7-9). This generates slight variations of many quantities throughout this paper. Sometimes we might deal with quantities like $z:=x+/ y-$. We cannot conclude directly that $z=(x / y)+$. In this case we create a variation of $y$ so 
small (compared to that of $x$ ) that we have $z=(x / y)+$. These details have been omitted for the sake of readability. We strongly recommend that the reader ignores these slight variations at the first reading.

\section{Proof of Theorem 1}

The proof relies upon Proposition 4 and the following lemma, which we prove on page 268 .

Lemma 6 (Local boundedness). Let $J=\left[t_{1}, t_{2}\right]$ be an interval. Assume that $u$ is a classical solution of (1-1). Let $Z(t):=\left\|\left(u(t), \partial_{t} u(t)\right)\right\|_{\tilde{H}^{2}\left(\mathbb{R}^{3}\right) \times H^{1}\left(\mathbb{R}^{3}\right)}$. There exists $0<\epsilon \ll$ constant such that if

$$
\|u\|_{L_{t}^{4} L_{x}^{12}\left(J \times \mathbb{R}^{3}\right)} \leq \frac{\epsilon}{g^{1 / 4}\left(Z\left(t_{1}\right)\right)},
$$

then there exists $C_{l}>0$ such that

$$
Z(t) \leq 2 C_{l} Z\left(t_{1}\right) \quad \text { for } t \in J
$$

We claim that the set

$$
\mathscr{F}:=\left\{T \in[0, \infty): \sup _{t \in[0, T]}\left\|\left(u(t), \partial_{t} u(t)\right)\right\|_{\tilde{H}^{2}\left(\mathbb{R}^{3}\right) \times H^{1}\left(\mathbb{R}^{3}\right)} \leq M_{0}\right\}
$$

is equal to $[0, \infty)$ for some constant $M_{0}:=M_{0}\left(\left\|u_{0}\right\|_{\tilde{H}^{2}\left(\mathbb{R}^{3}\right)},\left\|u_{1}\right\|_{H^{1}\left(\mathbb{R}^{3}\right)}\right)$ large enough. Indeed, $0 \in \mathscr{F}$ (this is clear); $\mathscr{F}$ is closed, by continuity; and $\mathscr{F}$ is open. To see this last fact, let $T \in \mathscr{F}$. Then by continuity there exists $\delta>0$ such that

$$
\sup _{t \in\left[0, T^{\prime}\right]}\left\|\left(u(t), \partial_{t} u(t)\right)\right\|_{H^{2}\left(\mathbb{R}^{3}\right) \times H^{1}\left(\mathbb{R}^{3}\right)} \leq 2 M_{0}
$$

for every $T^{\prime} \in[0, T+\delta)$. By (1-29) and (1-30) we have

$$
\|u\|_{L_{t}^{4} L_{x}^{12}\left(\left[0, T^{\prime}\right] \times \mathbb{R}^{3}\right)}^{4} \leq \max \left(C_{L, 0},\left(C_{L, 1} E g\left(2 M_{0}\right)\right)^{C_{L, 2}\left(E^{(193 / 4)+} g^{(225 / 8)+}\left(2 M_{0}\right)\right)}\right) .
$$

Let $N \geq 1$ and let $\underline{Z}(0):=\max (Z(0), 1)$. Without loss of generality we can assume that $C_{l} \gg 1$ so that $2 C_{l} \underline{Z}(0) \gg 1$ and $\log ^{c}\left(2 C_{l} \underline{Z}(0)\right) \gg 1$. We have, by the elementary rules of the logarithm and the inequality $\log ^{c}(2 n x) \leq \log ^{c}\left((2 n)^{x}\right)$ for $n \geq 1$ and $x \gg 1$ :

$$
\begin{aligned}
\sum_{n=1}^{N} \frac{\epsilon^{4}}{g\left(\left(2 C_{l}\right)^{n} Z_{0}\right)} & \geq \sum_{n=1}^{N} \frac{\epsilon^{4}}{\log ^{c}\left(\log \left(\left(2 C_{l}\right)^{2 n} \underline{Z}^{2 n}(0)+10\right)\right)} \gtrsim \sum_{n=1}^{N} \frac{1}{\log ^{c}\left(2 n \log \left(2 C_{l} \underline{Z}(0)\right)\right)} \\
& \gtrsim \frac{1}{\log ^{c}\left(2 C_{l} \underline{Z}(0)\right)} \sum_{n=1}^{N} \frac{1}{\log ^{c}(2 n)} \gtrsim \frac{1}{\log ^{c}\left(2 C_{l} \underline{Z}(0)\right)} \int_{1}^{N+1} \frac{1}{\log ^{c}(2 t)} d t \\
& \gtrsim \frac{1}{\log ^{c}\left(2 C_{l} \underline{Z}(0)\right)} \int_{1}^{N+1} \frac{1}{t^{1 / 2}} d t \gtrsim \frac{N^{1 / 2}}{\log ^{c}\left(2 C_{l} \underline{Z}(0)\right)} .
\end{aligned}
$$


By Lemma 6, (2-5) and (2-6) we can construct a partition $\left(J_{n}\right)_{1 \leq n \leq N}$ of $\left[0, T^{\prime}\right]$ such that

$$
\begin{array}{ll}
\|u\|_{L_{t}^{4} L_{x}^{12}\left(J_{n} \times \mathbb{R}^{3}\right)}=\frac{\epsilon}{g^{1 / 4}\left(\left(2 C_{l}\right)^{n} Z_{0}\right)}, & 1 \leq n<N, \\
\|u\|_{L_{t}^{4} L_{x}^{12}\left(J_{N} \times \mathbb{R}^{3}\right)} \leq \frac{\epsilon}{g^{1 / 4}\left(\left(2 C_{l}\right)^{N} Z_{0}\right)}, & Z(t) \leq\left(2 C_{l}\right)^{n} Z(0),
\end{array}
$$

for $t \in J_{1} \cup \cdots \cup J_{n}$ and

$$
\frac{N^{1 / 2}}{\log ^{c}\left(2 C_{l} \underline{Z}(0)\right)} \leq \max \left(C_{L, 0},\left(C_{L, 1} E g\left(2 M_{0}\right)\right)^{C_{L, 2}\left(E^{193 / 4+} g^{225 / 8+}\left(2 M_{0}\right)\right)}\right) .
$$

Since $c<8 / 225$ we have by $(1-24)$

$\log N \lesssim \log ^{c}\left(2 C_{l} \underline{Z}(0)\right)+\log \left(C_{L, 0}\right)$

$$
\begin{aligned}
& \quad+C_{L, 2} E^{(193 / 4)+} \log ^{(225 c / 8)+} \log \left(10+4 M_{0}^{2}\right) \log \left(C_{L, 1} E \log ^{c} \log \left(10+4 M_{0}^{2}\right)\right) \\
& \leq \log \left(\frac{\log \left(M_{0} / Z(0)\right)}{\log \left(2 C_{l}\right)}\right),
\end{aligned}
$$

if $M_{0}=M_{0}\left(\left\|u_{0}\right\|_{\tilde{H}^{2}\left(\mathbb{R}^{3}\right)},\left\|u_{1}\right\|_{H^{1}(\mathbb{R})}\right)$ is large enough. To prove the last inequality in (2-8) it is enough, by using (1-24), to notice that $\lim _{M_{0} \rightarrow \infty} f\left(M_{0}\right)=0$ with

$$
f\left(M_{0}\right):=\frac{\log ^{c}\left(2 C_{l} \underline{Z}(0)\right)+\log \left(C_{L, 0}\right)+C_{L, 2} E^{(193 / 4)+} \log ^{(225 c / 8)+} \log \left(10+4 M_{0}^{2}\right) \log \left(C_{L, 1} E \log ^{c} \log \left(10+4 M_{0}^{2}\right)\right)}{\log \left(\frac{\log \left(M_{0} / Z(0)\right)}{\log \left(2 C_{l}\right)}\right)} .
$$

Therefore we conclude that

$$
\sup _{t \in\left[0, T^{\prime}\right]}\left\|\left(u(t), \partial_{t} u(t)\right)\right\|_{H^{2}\left(\mathbb{R}^{3}\right) \times H^{1}\left(\mathbb{R}^{3}\right)} \leq\left(2 C_{l}\right)^{N} Z(0) \leq M_{0}
$$

Proof of Lemma 6. By the Strichartz estimates (1-16), the Sobolev embeddings (1-22) and (1-23) and the elementary estimate $\left|u^{5} \nabla(g(u))\right| \lesssim\left|u^{4} \nabla u g(u)\right|$, we have

$$
\begin{aligned}
Z(t) & \lesssim Z\left(t_{1}\right)+\left\|u^{5} g(u)\right\|_{L_{t}^{1} L_{x}^{2}\left(\left[t_{1}, t\right] \times \mathbb{R}^{3}\right)}+\left\|u^{4} \nabla u g(u)\right\|_{L_{t}^{1} L_{x}^{2}\left(\left[t_{1}, t\right] \times \mathbb{R}^{3}\right)}+\left\|u^{5} \nabla(g(u))\right\|_{L_{t}^{1} L_{x}^{2}\left(\left[t_{1}, t\right] \times \mathbb{R}^{3}\right)} \\
& \lesssim Z\left(t_{1}\right)+\left\|u^{5} g(u)\right\|_{L_{t}^{1} L_{x}^{2}\left(\left[t_{1}, t\right] \times \mathbb{R}^{3}\right)}+\left\|u^{4} \nabla u g(u)\right\|_{L_{t}^{1} L_{x}^{2}\left(\left[t_{1}, t\right] \times \mathbb{R}^{3}\right)} \\
& \lesssim Z\left(t_{1}\right)+\|u\|_{L_{t}^{4} L_{x}^{12}\left(\left[t_{1}, t\right] \times \mathbb{R}^{3}\right)}^{4}\|u\|_{L_{t}^{\infty} L_{x}^{6}\left(\left[t_{1}, t\right] \times \mathbb{R}^{3}\right)} g\left(\|u\|_{L_{t}^{\infty} L_{x}^{\infty}\left(\left[t_{1}, t\right] \times \mathbb{R}^{3}\right)}\right) \\
& \quad+\|u\|_{L_{t}^{4} L_{x}^{12}\left(\left[t_{1}, t\right] \times \mathbb{R}^{3}\right)}^{4}\|\nabla u\|_{L_{t}^{\infty} L_{x}^{6}\left(\left[t_{1}, t\right] \times \mathbb{R}^{3}\right)} g\left(\|u\|_{L_{t}^{\infty} L_{x}^{\infty}\left(\left[t_{1}, t\right] \times \mathbb{R}^{3}\right)}\right) \\
& \lesssim Z\left(t_{1}\right)+\|u\|_{L_{t}^{4} L_{x}^{12}\left(\left[t_{1}, t\right] \times \mathbb{R}^{3}\right)}^{4} Z(t) g(Z(t))
\end{aligned}
$$

Let $C_{l}$ be the constant determined by the last inequality in (2-11). From (2-1), (2-11) and a continuity argument, we have (2-2).

\section{Proof of Proposition 4}

The proof relies upon five lemmas, which we state here and then prove in subsequent sections, after seeing how they imply the proposition. 
Lemma 7 (Long time estimate if energy small). Let $J=\left[t_{1}, t_{2}\right]$ be a time interval. Let $u$ be a classical solution of (1-1). Assume that (1-28) holds. If

$$
E \ll \frac{1}{g^{1 / 2}(M)},
$$

then

$$
\|u\|_{L_{t}^{4} L_{x}^{12}\left(J \times \mathbb{R}^{3}\right)} \lesssim 1 .
$$

Lemma 8 (If $\|u\|_{L_{t}^{4} L_{x}^{12}\left(J \times \mathbb{R}^{3}\right)}$ is nonnegligible a mass concentration bubble exists and the size of $J$ is bounded from below). Let $u$ be a classical solution of (1-1). Let $J$ be a time interval. Assume that (1-28) holds. Let $\eta$ be a positive number such that

If $\|u\|_{L_{t}^{4} L_{x}^{12}\left(J \times \mathbb{R}^{3}\right)} \geq \eta$, then

$$
\eta \leq \frac{E^{1 / 12}}{g^{5 / 24}(M)}
$$

$$
\|u\|_{L_{t}^{\infty} L_{x}^{6}\left(J \times \mathbb{R}^{3}\right)} \gtrsim \eta^{2+} E^{-((1 / 2)+)} .
$$

Moreover, there exist a point $x_{0} \in \mathbb{R}^{3}$, a time $t_{0} \in J$ and a positive number $r$ such that we have the mass concentration estimate in the ball $B\left(x_{0}, r\right)$

$$
\int_{B\left(x_{0}, r\right)}\left|u\left(t_{0}, y\right)\right|^{2} d y \gtrsim \eta^{6+} E^{-(2+)} r^{2},
$$

and the following lower bound on the size of $J$ :

$$
|J| \gtrsim \eta^{4} E^{-2 / 3} r .
$$

Lemma 9 (Potential energy decay estimate). Let $u$ be a classical solution of (1-1). Let $[a, b]$ be an interval. Then we have the potential energy decay estimate

$$
\int_{|x| \leq b} F(u(b, x)) d x \lesssim \frac{a}{b}\left(e(a)+e^{1 / 3}(a)\right)+e(b)-e(a)+(e(b)-e(a))^{1 / 3} .
$$

Lemma $10\left(L_{t}^{4} L_{x}^{12}\right.$ norm of $u$ is small on a large truncation of the forward light cone). Let $J=\left[t_{1}, t_{2}\right]$ be an interval. Let $u$ be a classical solution of (1-1). Assume that (1-28) holds. Let $\eta$ be a positive number such that

$$
\eta \ll \min \left(E^{1 / 4}, E^{5 / 18}, \frac{E^{1 / 12}}{g^{5 / 24}(M)}\right) .
$$

Assume also that there exists $C_{2} \gg 1$ such that

$$
\left[t_{1},\left(C_{2} E^{10+} \eta^{-(36+)}\right)^{4 C_{2} E^{10+} \eta^{-(36+)}} t_{1}\right] \subset J .
$$

Then there exists a subinterval $J^{\prime}=\left[t_{1}^{\prime}, t_{2}^{\prime}\right]$ such that $\left|t_{2}^{\prime} / t_{1}^{\prime}\right| \sim E^{10+} \eta^{-(36+)}$ and

$$
\|u\|_{L_{t}^{4} L_{x}^{12}\left(\Gamma_{+}\left(J^{\prime}\right)\right)} \leq \eta \text {. }
$$

Lemma 11 (Asymptotic stability). Let $J=\left[t_{1}, t_{2}\right]$ be a time interval. Let $J^{\prime}=\left[t_{1}^{\prime}, t_{2}^{\prime}\right] \subset J$ and let $t \in J / J^{\prime}$. Let $u$ be a classical solution of (1-1). Assume that (1-28) holds. Then

$$
\left\|u_{l, t_{2}^{\prime}}(t)-u_{l, t_{1}^{\prime}}(t)\right\|_{L_{x}^{\infty}\left(\mathbb{R}^{3}\right)} \lesssim \frac{E^{5 / 6} g^{1 / 6}(M)}{\operatorname{dist}^{1 / 2}\left(t, J^{\prime}\right)} .
$$


We are ready to prove Proposition 4. We assume that we have an a priori bound $M$ of the $L_{t}^{\infty} \tilde{H}^{2}\left(J \times \mathbb{R}^{3}\right)$ norm of the solution $u$. There are two steps:

- If $E \ll 1 / g^{1 / 2}(M)$, then we know from Lemma 7 that (1-29) holds.

- Therefore we assume that the energy is large, that is,

$$
E \gtrsim \frac{1}{g^{1 / 2}(M)}
$$

We can assume without loss of generality that

$$
\|u\|_{L_{t}^{4} L_{x}^{12}\left(J \times \mathbb{R}^{3}\right)} \geq \frac{E^{1 / 12}}{g^{5 / 24}(M)} .
$$

From (3-13) we can partition $J$ into subintervals $J_{1}, \ldots, J_{l}$ such that for $i=1, \ldots, l-1$,

$$
\|u\|_{L_{t}^{4} L_{x}^{12}\left(J_{i} \times \mathbb{R}^{3}\right)}=\frac{E^{1 / 12}}{g^{5 / 24}(M)} \quad \text { and } \quad\|u\|_{L_{t}^{4} L_{x}^{12}\left(J_{l} \times \mathbb{R}^{3}\right)} \leq \frac{E^{1 / 12}}{g^{5 / 24}(M)} .
$$

Before moving forward we say that an interval $J_{i}$ is exceptional if

$$
\left\|u_{l, t_{1}}\right\|_{L_{t}^{4} L_{x}^{12}\left(J_{i} \times \mathbb{R}^{3}\right)}+\left\|u_{l, t_{2}}\right\|_{L_{t}^{4} L_{x}^{12}\left(J_{i} \times \mathbb{R}^{3}\right)} \geq \frac{1}{\left(C_{3} E g(M)\right)^{C_{4}\left(E^{(193 / 4)+} g^{(225 / 8)+}(M)\right)}},
$$

for some $C_{3} \gg 1, C_{4} \gg 1$ to be chosen later. (The numbers $193 / 4$ and 225/8 will play an important role in (3-44).) Otherwise $J_{i}$ is unexceptional. Let $\mathscr{E}$ denote the set of $J_{i}^{\prime}$ s that are exceptional and let $\overline{\mathscr{E} c}$ denote the set of nonempty sequences of consecutive unexceptional intervals $J_{i}$. By (1-16), (3-12) and (3-15),

$$
\operatorname{card}(\mathscr{E}) \lesssim E^{2}[O(E g(M))]^{O\left(E^{(193 / 4)+} g^{(225 / 8)+}(M)\right)} \lesssim[O(E g(M))]^{O\left(E^{(193 / 4)+} g^{(225 / 8)+}(M)\right)} .
$$

Since card $(\overline{\mathscr{E} c}) \lesssim \operatorname{card}(\mathscr{E})$ we have

$$
\|u\|_{L_{t}^{4} L_{x}^{12}\left(J \times \mathbb{R}^{3}\right)}^{4} \lesssim[O(E g(M))]^{O\left(E^{(193 / 4)+} g^{(225 / 8)+}(M)\right)}\left(\frac{E^{1 / 3}}{g^{5 / 6}(M)}+\sup _{K \in \in_{\mathscr{G} c}}\|u\|_{L_{t}^{4} L_{x}^{12}\left(K \times \mathbb{R}^{3}\right)}^{4}\right) .
$$

Let $K=J_{i_{0}} \cup \cdots \cup J_{i_{1}}$ be a sequence of consecutive unexceptional intervals. If $N(K)$ is the number of $J_{i}$ s making $K$ then by (3-12), (3-14) and (3-17) we have

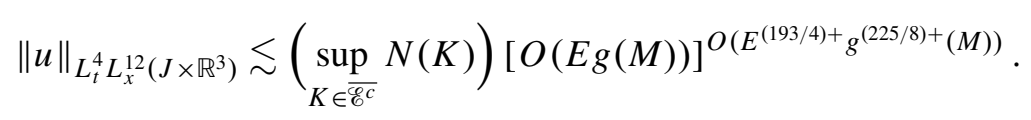

Therefore it suffices to estimate $N(K)$ for every $K=J_{i_{0}} \cup \cdots \cup J_{i_{1}}$. We will do that by first determining a lower bound for the size of the elements $J_{i}$ s and then by estimating the size of $K$. By (3-12), (3-14) and Lemma 8 , there exists for $i \in\left[i_{0}, \ldots i_{1}\right]$ a $\left(t_{i}, r_{i}, x_{i}\right) \in\left(J_{i} \times(0, \infty) \times \mathbb{R}^{3}\right)$ such that

$$
\frac{1}{r_{i}^{2}} \int_{B\left(x_{i}, r_{i}\right)}\left|u\left(t_{i}, y\right)\right|^{2} d y \gtrsim \frac{E^{-(3 / 2+)}}{g^{5 / 4+}(M)}
$$

and

$$
\left|J_{i}\right| \gtrsim \frac{E^{-1 / 3} r_{i}}{g^{5 / 6}(M)}
$$


Let $k \in\left[i_{0}, \ldots, i_{1}\right]$ be such that $r_{k}=\min _{i \in\left[i_{0}, i_{1}\right]} r_{i}$; let $f(t, r, x):=\frac{1}{r^{2}} \int_{B(x, r)}|u(t, y)|^{2} d y$; let $C_{5}$ be the constant determined by (3-19); and let $r_{0}=r_{0}(M)$ be defined by

$$
r_{0} M^{2}=\frac{C_{5} E^{-((3 / 2)+)}}{4 g^{(5 / 4)+}(M)} \text {. }
$$

Since $f(t, r, x) \leq r M^{2}$ we have

$$
f\left(t, r_{0}, x\right) \leq \frac{C_{5} E^{-((3 / 2)+)}}{4 g^{(5 / 4)+(M)}} .
$$

The set $A:=\left\{(t, r, x): t \in K, r_{0} \leq r \leq r_{k}, x \in \mathbb{R}^{3}\right\}$ is connected. Therefore its image is connected by $f$ and there exists $(\tilde{t}, \tilde{r}, \tilde{x}) \in K \times\left[r_{0}, r_{k}\right] \times \mathbb{R}^{3}$ such that $f(\tilde{t}, \tilde{r}, \tilde{x})=\left(C_{5} E^{-((3 / 2)+)}\right) /\left(2 g^{(5 / 4)+}(M)\right)$. In other words we have the following mass concentration

$$
\frac{1}{\tilde{r}^{2}} \int_{B(\tilde{x}, \tilde{r})} u^{2}(\tilde{t}, x) d x=\frac{C_{5} E^{-(3 / 2+)}}{2 g^{(5 / 4)+(M)}} .
$$

Moreover we have the useful lower bound for the size of $J_{i},{ }^{1} i_{0} \leq i \leq i_{1}$ :

$$
\left|J_{i}\right| \gtrsim \tilde{r} \frac{E^{-1 / 3}}{g^{5 / 6}(M)} .
$$

At this point we need to use the following lemma, which gives information about the size of $K$.

Lemma 12. Let $K$ be a sequence of unexceptional intervals. Assume there exist $\bar{t} \in K, \bar{x} \in \mathbb{R}^{3}$ and $\bar{r} \in(0, \infty)$ such that

$$
\frac{1}{\bar{r}^{2}} \int_{B(\bar{x}, \bar{r})} u^{2}(\bar{t}, y) d y \gtrsim E^{-((3 / 2)+)} g^{(5 / 4)+}(M) .
$$

Then there exist two constants $C_{6} \gg 1, C_{7} \gg 1$ such that

$$
|K| \leq\left(C_{6} E g(M)\right)^{C_{7} E^{(193 / 4)+} g^{(225 / 8)+}(M)} \bar{r} .
$$

If we combine the lemma with (3-22) we can estimate $N(K)$. More precisely, by Lemma $12,(3-22)$ and (3-12) we have

$$
N(K) \lesssim \frac{\left(C_{6} E g(M)\right)^{C_{7} E^{(193 / 4)+} g^{(225 / 8)+}(M)} \tilde{r}}{\tilde{r} \frac{E^{-(1 / 3)}}{g^{5 / 6}(M)}} \lesssim(O(E g(M)))^{O\left(E^{(193 / 4)+} g^{(225 / 8)+}(M)\right)}
$$

Plugging this upper bound for $N(K)$ into (3-18) we get (1-30), completing the proof of the proposition (modulo the lemmas).

Proof of Lemma 12. By using the space translation invariance of (1-1) we can reduce to the case where $\bar{x}$ vanishes. $^{2}$ By using the time reversal invariance and the time translation invariance ${ }^{3}$ it suffices to estimate $|K \cap[\bar{t}, \infty)|$. By using the time translation invariance again ${ }^{4}$ we can assume that $\bar{t}=\bar{r}$ and

${ }^{1}$ Notice that we have the lower bound $\tilde{r} \geq C_{5} E^{-((3 / 2)+)} /\left(4 M^{2} g^{(5 / 4)+}(M)\right)$. One might think that the presence of $\tilde{r}$ in (3-22) is annoying since this lower bound is crude. However we will see that $\tilde{r}$ disappears at the end of the process: see (3-25). Therefore a sharp lower bound is not required.

${ }^{2}$ We consider the function $u_{1}(t, x)=u(t, x-\bar{x})$ and we abuse notation in the sequel by writing $u_{1}$ for $u$.

${ }^{3}$ We consider the function $u_{2}(t, x):=u(2 \bar{t}-t, x)$ and we abuse notation in the sequel by writing $u_{2}$ for $u$.

${ }^{4}$ We consider the function $u_{3}(t, x):=u(t+(\bar{t}-\bar{r}), x)$ and we abuse notation in the sequel by writing $u_{3}$ for $u$. 
therefore $\bar{r} \in K$. Let $K_{+}:=K \cap[\bar{r}, \infty)$. We are interested in estimating $\left|K_{+}\right|$. We would like to use Lemma 10. Therefore, we consider the set $\Gamma_{+}\left(K_{+}\right)$. We have

$$
\frac{1}{\bar{r}^{2}} \int_{B(0, \bar{r})}|u(\bar{r}, y)|^{2} d y \gtrsim \frac{E^{-((3 / 2)+)}}{g^{(5 / 4)+(M)}} .
$$

Therefore by Proposition 3 and (3-26) we have

$$
\int_{B(0, \bar{r})}|u(t, y)|^{2} d y \gtrsim \frac{E^{-((3 / 2)+)} \bar{r}^{2}}{g^{(5 / 4)+}(M)}
$$

if $(t-\bar{r}) E^{1 / 2} \leq\left(c_{0} E^{-((3 / 4)+)} \bar{r} / g^{(5 / 8)+}(M)\right)$ for some $c_{0} \ll 1$. Therefore by Hölder there exists $0<c_{1} \ll 1$ small enough such that

$$
\|u\|_{L_{t}^{4} L_{x}^{12}}\left(\Gamma_{+}\left(\left[\bar{r}, \bar{r}+\frac{c_{0} E^{-((5 / 4)+)}}{g^{(5 / 8)+}(M)}\right]\right)\right) \geq c_{1} \frac{E^{-17 / 16}}{g^{25 / 32}(M)} .
$$

Suppose first that $\|u\|_{L_{t}^{4} L_{x}^{12}\left(\Gamma_{+}\left(K_{+}\right)\right)} \leq c_{1} \frac{E^{-(17 / 16)}}{g^{(25 / 32)}(M)}$. In this case we get from (3-28)

$$
K_{+} \subset\left[\bar{r}, \bar{r}+\frac{c_{0} E^{-((5 / 4)+)} \bar{r}}{g^{(5 / 8)+}(M)}\right],
$$

and, using also (3-12), we get (3-24).

Now suppose instead that $\|u\|_{L_{t}^{4} L_{x}^{12}\left(\Gamma_{+}\left(K_{+}\right)\right)} \geq c_{1} \frac{E^{-((17 / 16)+)}}{g^{(25 / 32)+}(M)}$. Define

$$
\tilde{\eta}:=\frac{c_{1}}{4} \frac{E^{-((17 / 16))+}}{g^{(25 / 32)+(M)}},
$$

and divide $\Gamma_{+}\left(K_{+}\right)$into consecutive cone truncations $\Gamma_{+}\left(\tilde{J}_{1}\right), \ldots, \Gamma_{+}\left(\tilde{J}_{k}\right)$ such that, for $j=1, \ldots, k-1$,

$$
\|u\|_{L_{t}^{4} L_{x}^{12}\left(\Gamma_{+}\left(\tilde{J}_{j}\right)\right)}=\tilde{\eta}
$$

and

$$
\|u\|_{L_{t}^{4} L_{x}^{12}\left(\Gamma_{+}\left(\tilde{J}_{k}\right)\right)} \leq \tilde{\eta} .
$$

We get from (3-28)

$$
\tilde{J}_{1} \subset\left[\bar{r}, \bar{r}+\frac{c_{0} E^{-((5 / 4)+)} \bar{r}}{g^{(5 / 8)+}(M)}\right] .
$$

Result 13. If $j \in[1, \ldots, k-1]$ we either have

$$
\left|\tilde{J}_{j+1}\right| \lesssim\left|\tilde{J}_{j}\right| \tilde{\eta}^{-4} E^{8 / 3} g^{1 / 3}(M)
$$

or

$$
\left|\tilde{J}_{j}\right| \geq\left(C_{6} E g(M)\right)^{C_{7} E^{(193 / 4)+} g^{(225 / 8)+}(M)} \bar{r}
$$

for some constants $C_{6} \gg 1, C_{7} \gg 1$. 
Proof. We get from (1-21), (3-12) and (3-30)

$$
\begin{aligned}
\left\|u-u_{l, t_{j+1}}\right\|_{L_{t}^{4} L_{x}^{12}\left(\Gamma_{+}\left(\tilde{J}_{j}\right)\right)} & \lesssim\left\|u^{5} g(u)\right\|_{L_{t}^{1} L_{x}^{2}\left(\Gamma_{+}\left(\tilde{J}_{j} \cup \tilde{J_{j+1}}\right)\right)} \\
& \lesssim\left\|u^{4}\right\|_{L_{t}^{1} L_{x}^{3}\left(\Gamma_{+}\left(\tilde{J} \cup \tilde{j_{j+1}}\right)\right)}\left\|u g^{1 / 6}(u)\right\|_{L_{t}^{\infty} L_{x}^{6}\left(\Gamma _ { + } \left(\tilde{\left.\left.J_{j} \cup \tilde{j}_{j+1}\right)\right)} g^{5 / 6}(M)\right.\right.} \\
& \lesssim \tilde{\eta}^{4} E^{1 / 6} g^{5 / 6}(M) \\
& \ll \tilde{\eta},
\end{aligned}
$$

with $J_{j}=\left[t_{j-1}, t_{j}\right]$. Therefore by (3-31) we have $\left\|u_{l, t_{j+1}}\right\|_{L_{t}^{4} L_{x}^{12}\left(\Gamma_{+}\left(\tilde{J}_{j}\right)\right)} \sim \tilde{\eta}$. This implies that

$$
\left\|u_{l, t_{j+1}}-u_{l, t_{2}}\right\|_{L_{t}^{4} L_{x}^{12}\left(\Gamma_{+}\left(\tilde{J}_{j}\right)\right)} \gtrsim \tilde{\eta}
$$

or

$$
\left\|u_{l, t_{2}}\right\|_{L_{t}^{4} L_{x}^{12}\left(\Gamma_{+}\left(\tilde{J}_{j}\right)\right)} \gtrsim \tilde{\eta} .
$$

Case 1. $\left\|u_{l, t_{j+1}}-u_{l, t_{2}}\right\|_{L_{t}^{4} L_{x}^{12}\left(\Gamma_{+}\left(\tilde{J}_{i}\right)\right)} \gtrsim \tilde{\eta}$. By Lemma 11 and Hölder we have

$$
\begin{aligned}
\left\|u_{l, t_{j+1}}-u_{l, t_{2}}\right\|_{L_{t}^{4} L_{x}^{12}\left(\Gamma_{+}\left(\tilde{J}_{j}\right)\right)} & \lesssim\left|\tilde{J}_{j}\right|^{1 / 4}\left\|u_{l, t_{j+1}}-u_{l, t_{2}}\right\|_{L_{t}^{\infty} L_{x}^{12}\left(\Gamma_{+}\left(\tilde{J}_{j}\right)\right)} \\
& \lesssim\left|\tilde{J}_{j}\right|^{1 / 4}\left\|u_{l, t_{j+1}}-u_{l, t_{2}}\right\|_{L_{t}^{\infty} L_{x}^{\infty}\left(\Gamma_{+}\left(\tilde{J}_{j}\right)\right)}^{1 / 2}\left\|u_{l, t_{j+1}}-u_{l, t_{2}}\right\|_{L_{t}^{\infty} L_{x}^{6}\left(\Gamma_{+}\left(\tilde{J}_{j}\right)\right)}^{1 / 2} \\
& \lesssim \frac{\left|\tilde{J}_{j}\right|^{1 / 4} E^{2 / 3} g^{1 / 12}(M)}{\left|\tilde{J}_{j+1}\right|^{1 / 4}} .
\end{aligned}
$$

We get (3-34) from (3-37) and (3-39).

Case 2. $\left\|u_{l, t_{2}}\right\|_{L_{t}^{4} L_{x}^{12}\left(\Gamma_{+}\left(\tilde{J}_{j}\right)\right)} \gtrsim \tilde{\eta}$. In this case $\left\|u_{l, t_{2}}\right\|_{L_{t}^{4} L_{x}^{12}\left(\tilde{J}_{j}\right)} \gtrsim \tilde{\eta}$. Recall that $K_{+}$is a subinterval of $K=J_{i_{0}} \cup \cdots \cup J_{i_{1}}$, sequence of unexceptional intervals $J_{i}, i_{0} \leq i \leq i_{1}$. Consequently there are at least $\sim \tilde{\eta}\left(C_{3} E g(M)\right)^{C_{4} E^{(193 / 4)+} g^{(225 / 8)+}(M)}$ intervals $J_{j}$ that cover $\tilde{J}_{i}$. Therefore we get (3-35) from (3-22) and (3-12).

Using Result 13 and Lemma 10 we can get an upper bound on the size $\left|K_{+}\right|$:

Result 14. We have

$$
\left|K_{+}\right| \leq\left(C_{6} E g(M)\right)^{C_{7}\left(E^{(193 / 4)+} g^{(225 / 8)+}(M)\right)} \bar{r} .
$$

Proof. Let $B:=\left(C_{6} E g(M)\right)^{C_{7}\left(E^{(193 / 4)+} g^{(225 / 8)+}(M)\right)}$. Assume that (3-40) fails. Let $\tilde{J}_{j_{1}}$ be the first interval for which $\left|\tilde{J}_{1} \cup \cdots \cup \tilde{J}_{j_{1}}\right|$ exceeds $B \bar{r}$. Then $j_{1} \neq 1,\left|\tilde{J}_{j_{1}}\right| \lesssim\left|\tilde{J}_{j_{1}-1}\right| \tilde{\eta}^{-4} E^{8 / 3} g^{1 / 3}(M)$ and we have

$$
\frac{c_{1} E^{-5 / 4} \tilde{r}}{g^{(5 / 8)}(M)}+T_{2}-T_{1}+\left(T_{2}-T_{1}\right) \tilde{\eta}^{-4} E^{8 / 3} g^{1 / 3}(M) \gtrsim\left|\tilde{J}_{1}\right|+\cdots+\left|\tilde{J}_{j_{1}}\right| \geq B \bar{r},
$$

if $\left[T_{1}, T_{2}\right]:=\tilde{J}_{2} \cup \cdots \cup \tilde{J}_{j_{1}-1}$. Therefore by (3-12) and (3-41) we have

$$
T_{2}-T_{1} \gtrsim \frac{\tilde{\eta}^{4} E^{-(8 / 3)} B \bar{r}}{g^{1 / 3}(M)} .
$$

Moreover $T_{1} \leq \bar{r}+\left(c_{1} E^{-((5 / 4)+)} \bar{r}\right) /\left(g^{(5 / 8)+}(M)\right)$. Therefore by (3-12) we have

$$
T_{1}=O(\bar{r}) .
$$


By (3-42) and (3-43) we have

$$
\frac{T_{2}}{T_{1}} \geq\left(C_{2} E^{10+}\left(\frac{\tilde{\eta}}{4}\right)^{-(36+)}\right)^{4 C_{2} E^{10+}(\tilde{\eta} / 4)^{-(36+)}}
$$

with $C_{2}$ defined in Lemma 10, provided that $C_{6}, C_{7} \gg \max \left(c_{1}, C_{2}\right)$. Therefore we can apply Lemma 10 and find a subinterval $\left[t_{1}^{\prime}, t_{2}^{\prime}\right] \subset \tilde{J}_{2} \cup \cdots \cup \tilde{J}_{j_{1}-1}$ with $\left|t_{2}^{\prime} / t_{1}^{\prime}\right| \sim E^{10+} \tilde{\eta}^{-(36+)}$ and $\|u\|_{L_{t}^{4} L_{x}^{12}\left(\left[t_{1}^{\prime}, t_{2}^{\prime}\right]\right)} \leq \tilde{\eta} / 4$. This means that $\left[t_{1}^{\prime}, t_{2}^{\prime}\right] \subset\left[T_{1}, T_{2}\right]$ is covered by at most two consecutive intervals. It is convenient to introduce $\left[t_{1}^{\prime}, t_{2}^{\prime}\right]_{g}$, the geometric mean of $t_{1}^{\prime}$ and $t_{2}^{\prime}$. We have $\left[t_{1}^{\prime}, t_{2}^{\prime}\right]_{g} \sim \tilde{\eta}^{-18} E^{5} t_{1}^{\prime}$. There are two cases.

Case 1. $\left[t_{1}^{\prime}, t_{2}^{\prime}\right]$ is covered by one interval $\tilde{J}_{\bar{j}}=\left[a_{\bar{j}}, b_{\bar{j}}\right], 2 \leq \bar{j} \leq j_{1}-1$. Then $\left|\tilde{J}_{\bar{j}}\right| \gtrsim \tilde{\eta}^{-(36+)} E^{10+} t_{1}^{\prime}$ and $\left|\tilde{J}_{\bar{j}-1}\right| \leq t_{1}^{\prime}$. Therefore $\left|\tilde{J}_{\bar{j}}\right| \gtrsim \tilde{\eta}^{-(36+)} E^{10+}\left|\tilde{J}_{\bar{j}-1}\right|$. Contradiction with (3-12) and (3-34).

Case 2. $\left[t_{1}^{\prime}, t_{2}^{\prime}\right]$ is covered by two intervals $\tilde{J}_{\bar{j}}=\left[a_{\bar{j}}, b_{\bar{j}}\right]$ and $\tilde{J}_{\bar{j}+1}=\left[a_{\bar{j}+1}, b_{\bar{j}+1}\right]$ for some $2 \leq \bar{j} \leq j_{1}-2$. Then there are two subcases.

Case $2 a . b_{\bar{j}} \leq\left[t_{1}^{\prime}, t_{2}^{\prime}\right]_{g}$. In this case $\left|\tilde{J}_{\bar{j}+1}\right| \gtrsim \tilde{\eta}^{-(36+)} E^{10+} t_{1}^{\prime}$ and $\left|\tilde{J}_{\bar{j}}\right| \leq \tilde{\eta}^{-(18+)} E^{5+} t_{1}^{\prime}$. Therefore by (3-12) we have $\left|\tilde{J}_{\bar{j}+1}\right| \gtrsim \tilde{\eta}^{-(18+)} E^{5+}\left|\tilde{J}_{\bar{j}}\right|$. Contradiction with (3-12) and (3-34).

Case $2 b . \quad b_{\bar{j}} \geq\left[t_{1}^{\prime}, t_{2}^{\prime}\right]_{g}$. In this case by (3-12) $\left|\tilde{J}_{\bar{j}}\right| \gtrsim \tilde{\eta}^{-(18+)} E^{5+} t_{1}^{\prime}$ and $\left|\tilde{J}_{\bar{j}-1}\right| \leq t_{1}^{\prime}$. Therefore $\left|\tilde{J}_{\bar{j}}\right| \gtrsim \tilde{\eta}^{-(18+)} E^{5+}\left|\tilde{J}_{\bar{j}-1}\right|$. Contradiction with (3-12) and (3-34).

This exhausts all cases. Thus we have proved Result 14 and so also Lemma 12.

Remark 15. It seems likely that we can find a better upper bound for $\left|K_{+}\right|$than (3-40) by exploiting Lemma 11 in a better way. For instance we can consider $k$ successive time intervals $\tilde{J}_{j+1}, \ldots, \tilde{J}_{j+k}$, $k>1$ and prove an estimate like

$$
\left|\tilde{J}_{j+1}\right|+\cdots\left|\tilde{J}_{j+k}\right| \lesssim\left|\tilde{J}_{j}\right| \tilde{\eta}^{-4} E^{8 / 3} g^{1 / 3}(M)
$$

This estimate is stronger than (3-34). We can probably find a smaller $B$ such that (3-44) holds with $\tilde{\eta}$ substituted for something like $k \tilde{\eta}$ and, by modifying the argument above, find a contradiction with (3-45). At the end of the process we can probably prove global existence of smooth solutions to (1-1) for $0<c<c_{0}$, with $c_{0}>8 / 225$ to be determined. We will not pursue these matters.

\section{Proof of Lemma 7}

Applying the Strichartz estimates and the Hölder inequality,

$$
\begin{aligned}
\|u\|_{L_{t}^{4} L_{x}^{12}\left(J \times \mathbb{R}^{3}\right)} & \lesssim E^{1 / 2}+\left\|u^{4}\right\|_{L_{t}^{1} L_{x}^{2}\left(J \times \mathbb{R}^{3}\right)}\left\|u g^{1 / 6}(u)\right\|_{L_{t}^{\infty} L_{x}^{6}\left(J \times \mathbb{R}^{3}\right)}\left\|g^{5 / 6}(u)\right\|_{L_{t}^{\infty} L_{x}^{\infty}\left(J \times \mathbb{R}^{3}\right)} \\
& \lesssim E^{1 / 2}+E^{1 / 6} g^{5 / 6}(M)\|u\|_{L_{t}^{4} L_{x}^{12}\left(J \times \mathbb{R}^{3}\right)}^{4} .
\end{aligned}
$$

Hence (3-2) by (3-1) and a continuity argument. 


\section{Proof of Lemma 8}

Let $J^{\prime}=\left[t_{1}^{\prime}, t_{2}^{\prime}\right] \subset J$ be such that $\|u\|_{L_{t}^{4} L_{x}^{12}\left(J^{\prime} \times \mathbb{R}^{3}\right)}=\eta$. Then by (1-22) and (3-3)

$$
\begin{aligned}
\|f(u)\|_{L_{t}^{1} L_{x}^{2}\left(J^{\prime} \times \mathbb{R}^{3}\right)} & \lesssim\left\|u g^{1 / 6}(u)\right\|_{L_{t}^{\infty} L_{x}^{6}\left(J^{\prime} \times \mathbb{R}^{3}\right)}\|u\|_{L_{t}^{4} L_{x}^{12}\left(J^{\prime} \times \mathbb{R}^{3}\right)}^{4}\left\|g^{5 / 6}(u)\right\|_{L_{t}^{\infty} L_{x}^{\infty}\left(J^{\prime} \times \mathbb{R}^{3}\right)} \\
& \lesssim E^{1 / 6} \eta^{4} g^{5 / 6}(M) \lesssim E^{1 / 2}
\end{aligned}
$$

It is slightly unfortunate that $(2, \infty)$ is not wave admissible. Therefore we consider the admissible pair $(2+\epsilon, 6(2+\epsilon) / \epsilon)$ with $\epsilon \ll 1$. By the Strichartz estimates and (5-1), we have

$$
\|u\|_{L_{t}^{2+\epsilon} L_{x}^{(6(2+\epsilon)) / \epsilon}\left(J^{\prime} \times \mathbb{R}^{3}\right)} \lesssim\left\|\nabla u\left(t_{1}^{\prime}\right)\right\|_{L^{2}\left(\mathbb{R}^{3}\right)}+\left\|u\left(t_{1}^{\prime}\right)\right\|_{L^{2}\left(\mathbb{R}^{3}\right)}+\|f(u)\|_{L_{t}^{1} L_{x}^{2}\left(J^{\prime} \times \mathbb{R}^{3}\right)} \lesssim E^{1 / 2} .
$$

Let $N$ be a frequency to be chosen later. By the Bernstein inequality and (1-7) we have

$$
\left\|P_{<N} u\right\|_{L_{t}^{4} L_{x}^{12}\left(J^{\prime} \times \mathbb{R}^{3}\right)} \lesssim N^{1 / 4}\left|J^{\prime}\right|^{1 / 4}\|u\|_{L_{t}^{\infty} L_{x}^{6}\left(J^{\prime} \times \mathbb{R}^{3}\right)} \lesssim N^{1 / 4}\left|J^{\prime}\right|^{1 / 4} E^{1 / 6} .
$$

Therefore

$$
\left\|P_{<N} u\right\|_{L_{t}^{4} L_{x}^{12}\left(J^{\prime} \times \mathbb{R}^{3}\right)} \lesssim\left|J^{\prime}\right|^{1 / 4} N^{1 / 4} E^{1 / 6} .
$$

Let $c_{2} \ll 1$. Then if $N=c_{2}^{4}\left(\eta^{4} /\left(\left|J^{\prime}\right| E^{2 / 3}\right)\right)$ we have

$$
\left\|P_{\geq N} u\right\|_{L_{t}^{4} L_{x}^{12}\left(J^{\prime} \times \mathbb{R}^{3}\right)} \gtrsim \eta \quad \text { and } \quad\|u\|_{L_{t}^{4} L_{x}^{12}\left(J^{\prime} \times \mathbb{R}^{3}\right)} \sim\left\|P_{\geq N} u\right\|_{L_{t}^{4} L_{x}^{12}\left(J^{\prime} \times \mathbb{R}^{3}\right)} .
$$

By (5-2) and (5-5) we have

$$
\begin{aligned}
\eta & \sim\left\|P_{\geq N} u\right\|_{L_{t}^{4} L_{x}^{12}\left(J^{\prime} \times \mathbb{R}^{3}\right)} \\
& \lesssim\left\|P_{\geq N} u\right\|_{L_{t}^{2+\epsilon} L_{x}^{(2+(2+\epsilon)) / \epsilon}\left(J^{\prime} \times \mathbb{R}^{3}\right)}^{(2+\epsilon) / 4}\left\|P_{\geq N} u\right\|_{L_{t}^{\infty} L_{x}^{6}\left(J^{\prime} \times \mathbb{R}^{3}\right)}^{1-(2+\epsilon) / 4} \\
& \lesssim E^{(2+\epsilon) / 8}\left\|P_{\geq N} u\right\|_{L_{t}^{\infty} L_{x}^{6}\left(J^{\prime} \times \mathbb{R}^{3}\right)}^{1-((2+\epsilon) / 4)} .
\end{aligned}
$$

Therefore we conclude that $\left\|P_{\geq N}\right\|_{L_{t}^{\infty} L_{x}^{6}\left(J^{\prime} \times \mathbb{R}^{3}\right)} \gtrsim \eta^{2+} E^{-((1 / 2)+)}$. Applying Proposition 2 we get (3-5).

\section{Proof of Lemma 9}

Bahouri and Gerard [1999, page 171] used arguments from Grillakis [1990; 1992] and Shatah-Struwe [1993] to derive an a priori estimate of the solution $u$ to the 3D quintic defocusing wave equation, that is, $\partial_{t t} u-\Delta u+u^{5}=0$. More precisely they were able to prove

$$
\int_{|x| \leq b}|u(b, x)|^{6} d x \lesssim \frac{a}{b}\left(\tilde{e}(a)+\tilde{e}^{1 / 3}(a)\right)+\tilde{e}(b)-\tilde{e}(a)+(\tilde{e}(b)-\tilde{e}(a))^{1 / 3},
$$

with

$$
\tilde{e}(t):=\frac{1}{2} \int_{|x| \leq t}\left(\partial_{t} u\right)^{2} d x+\frac{1}{2} \int_{|x| \leq t}|\nabla u|^{2} d x+\frac{1}{6} \int_{|x| \leq t} u^{6} d x .
$$

Since we apply their ideas to the potential $f$ we just sketch the proof. Given the cone $\Gamma_{+}([a, b])$ we denote by $\partial \Gamma_{+}([a, b])$ the mantle of the cone $\Gamma_{+}([a, b])$, that is,

$$
\partial \Gamma_{+}([a, b]):=\left\{\left(t^{\prime}, x\right) \in[a, b] \times \mathbb{R}^{3}, t=|x|\right\} .
$$


The local energy identity

$$
e(b)-e(a)=\frac{1}{2 \sqrt{2}} \int_{\partial \Gamma_{+}([a, b])}\left|\frac{x \partial_{t} u}{t}+\nabla u\right|^{2}+\frac{1}{\sqrt{2}} \int_{\partial \Gamma_{+}([a, b])} F(u)
$$

results from the integration of the identity $\partial_{t} u\left(\partial_{t t} u-\Delta u+f(u)\right)=0$ on the cone $\Gamma_{+}([a, b])$. We have [Shatah and Struwe 1998]

$$
\begin{aligned}
& \partial_{t}\left(\frac{t}{2}\left(\partial_{t} u\right)^{2}+\frac{t}{2}|\nabla u|^{2}+(x . \nabla u) \partial_{t} u+t F(u)+u \partial_{t} u\right) \\
& \quad-\operatorname{div}\left(t \nabla u \partial_{t} u+(x . \nabla u) \nabla u-\frac{|\nabla u|^{2} x}{2}+\frac{\left(\partial_{t} u\right)^{2} x}{2}-x F(u)+u \nabla u\right)+u f(u)-4 F(u)=0 .
\end{aligned}
$$

Integrating this identity on $\Gamma_{+}([a, b])$, we have

with

$$
X(b)-X(a)+Y(a, b)=\int_{\Gamma_{+}([a, b])} 4 F(u)-u f(u),
$$

$$
X(t):=\int_{|x| \leq t} \frac{t}{2}\left(\partial_{t} u\right)^{2}+\frac{t}{2}|\nabla u|^{2}+(x . \nabla u) \partial_{t} u+t F(u)+u \partial_{t} u
$$

and

$Y(a, b):=$

$$
\begin{aligned}
-\frac{1}{\sqrt{2}} \int_{\partial \Gamma_{+}([a, b])}\left(\frac{t}{2}\left(\partial_{t} u\right)^{2}+\frac{t}{2}|\nabla u|^{2}+(x . \nabla u) \partial_{t} u\right. & +t F(u)+u \partial_{t} u+t \frac{\nabla u \cdot x}{|x|} \partial_{t} u+\frac{|x \cdot \nabla u|^{2}}{|x|} \\
& \left.-\frac{|\nabla u|^{2}}{2}|x|+\frac{\left(\partial_{t} u\right)^{2}|x|}{2}-|x| F(u)+u \frac{\nabla u \cdot x}{|x|}\right) .
\end{aligned}
$$

In fact we have [Shatah and Struwe 1993]

$$
X(t)=\int_{|x| \leq t} t\left[\frac{1}{2}\left(\partial_{t} u\right)^{2}+\frac{1}{2}\left|\nabla u+\frac{u x}{|x|^{2}}\right|^{2}\right]+\partial_{t} u(x . \nabla u+u)+t F(u)-\int_{|x|=t} \frac{u^{2}}{2} .
$$

Since $t=|x|$ on $\partial \Gamma_{+}([a, b])$ we have

$$
Y(a, b)=-\frac{1}{\sqrt{2}} \int_{\partial \Gamma_{+}([a, b])}|x|\left(\partial_{t} u\right)^{2}+2(x . \nabla u) \partial_{t} u+u \partial_{t} u+\frac{(x . \nabla u)^{2}}{|x|}+u \frac{\nabla u \cdot x}{|x|},
$$

and after some computations [Shatah and Struwe 1993], we get

$$
Y(a, b)=-\frac{1}{\sqrt{2}} \int_{\partial \Gamma_{+}([a, b])} \frac{1}{t}\left(t \partial_{t} u+(\nabla u \cdot x)+u\right)^{2}+\int_{|x|=b} \frac{u^{2}}{2}-\int_{|x|=a} \frac{u^{2}}{2} .
$$

Therefore, if

$$
H(t):=\int_{|x| \leq t} t\left[\frac{1}{2}\left(\partial_{t} u\right)^{2}+\frac{1}{2}\left|\nabla u+\frac{u x}{|x|^{2}}\right|^{2}\right]+\partial_{t} u(x . \nabla u+u)+t F(u),
$$

then

$$
H(b)-H(a)=\frac{1}{\sqrt{2}} \int_{\partial \Gamma_{+}([a, b])} \frac{1}{t}\left(t \partial_{t} u+\nabla u \cdot x+u\right)^{2}+\int_{\Gamma_{+}([a, b])} 4 F(u)-u f(u) .
$$


We estimate $H(t)$, following [Bahouri and Gérard 1999]. We have

$$
\left|\partial_{t} u(x . \nabla u+u)\right| \leq \frac{t}{2}\left(\left(\partial_{t} u\right)^{2}+\left|\nabla u+\frac{u x}{|x|^{2}}\right|^{2}\right) \lesssim t\left(\left(\partial_{t} u\right)^{2}+|\nabla u|^{2}+\frac{u^{2}}{|x|^{2}}\right) .
$$

Therefore by (6-14), the Hölder inequality and (1-7), we have

$$
H(t) \lesssim t\left(e(t)+\int_{|x| \leq t} \frac{u^{2}}{|x|^{2}}\right) \lesssim t\left(e(t)+\left(\int_{|x| \leq t} u^{6}\right)^{1 / 3}\right) \lesssim t\left(e(t)+e^{1 / 3}(t)\right)
$$

Moreover by (6-4), the Hölder inequality and (1-7), we have

$$
\begin{aligned}
\frac{1}{\sqrt{2}} \int_{\partial \Gamma_{+}([a, b])} \frac{1}{t}\left(t \partial_{t} u+\nabla u \cdot x+u\right)^{2} & \lesssim \frac{b}{2 \sqrt{2}} \int_{\partial \Gamma_{+}([a, b])}\left(\frac{\nabla u \cdot x}{t}+\partial_{t} u\right)^{2}+\frac{1}{2 \sqrt{2}} \int_{\partial \Gamma_{+}([a, b])} \frac{u^{2}}{t^{2}} \\
& \lesssim b \int_{\partial \Gamma_{+}([a, b])}\left|\frac{x}{t} \partial_{t} u+\nabla u\right|^{2}+\frac{1}{2 \sqrt{2}}\left(\int_{\partial \Gamma_{+}([a, b])} u^{6}\right)^{1 / 3} \\
& \lesssim b\left((e(b)-e(a))+(e(b)-e(a))^{1 / 3}\right) .
\end{aligned}
$$

We get from (1-7)

$$
4 F(u)-u f(u) \leq 0
$$

By (6-13), and (6-15)-(6-17), we have

$$
\begin{aligned}
\int_{|x| \leq b} F(u) & \lesssim \frac{H(b)}{b} \lesssim \frac{H(a)+\frac{1}{\sqrt{2}} \int_{\partial \Gamma_{+}([a, b])} \frac{1}{t}\left(t \partial_{t} u+\nabla u \cdot x+u\right)^{2}}{b} \\
& \lesssim \frac{a}{b}\left(e(a)+e^{1 / 3}(a)\right)+e(b)-e(a)+(e(b)-e(a))^{1 / 3}
\end{aligned}
$$

\section{Proof of Lemma 10}

The proof relies upon two results that we prove in the subsections.

Result 16. Let $u$ be a classical solution of (1-1). Assume that (1-28) holds. Let $\eta$ be a positive number such that (3-3) holds. If $\|u\|_{L_{t}^{4} L_{x}^{12}\left(\Gamma_{+}(J)\right)} \geq \eta$ then

$$
\|u\|_{L_{t}^{\infty} L_{x}^{6}\left(\Gamma_{+}(J)\right)} \gtrsim \eta^{2+} E^{-((1 / 2)+)} .
$$

Result 17. Let $u$ be a smooth solution to (1-1). Assume that (1-28) holds. Let $\eta$ be a positive number such that

$$
\eta \leq \min \left(1, E^{1 / 18}\right)
$$

Let $J=\left[t_{1}, t_{2}\right]$ be an interval such that $\left[t_{1}, t_{1}\left(E \eta^{-18}\right)^{4 E \eta^{-18}}\right] \subset J$. Then there exists a subinterval $J^{\prime}=\left[t_{1}^{\prime}, t_{2}^{\prime}\right]$ such that $\left|t_{2}^{\prime} / t_{1}^{\prime}\right|=E \eta^{-18}$ and

$$
\|u\|_{L_{t}^{\infty} L_{x}^{6}\left(\Gamma_{+}\left(J^{\prime}\right)\right)} \lesssim \eta
$$


Let $C_{9}$ be the constant determined by $\gtrsim$ in (7-1). Let $C_{10}$ be the constant determined by $\lesssim$ in (7-3). We get from (3-9):

$$
\begin{aligned}
& {\left[t_{1}, t_{1}\left(E\left(\frac{C_{9} \eta^{2+} E^{-(1 / 2)+}}{2 C_{10}}\right)^{-18}\right)^{4 E\left(\frac{C_{9} \eta^{2+} E^{-(1 / 2)+}}{2 C_{10}}\right)^{-18}}\right] \subset\left[t_{1}, C_{2}\left(E^{10+} \eta^{-(36+)}\right)^{4 C_{2} E^{10+} \eta^{-(36+)}} t_{1}\right]} \\
& \subset J \text {, }
\end{aligned}
$$

if $C_{2} \gg \max \left(C_{9}, C_{10}\right)$. Therefore, since $\left(C_{9} \eta^{2+} E^{-(1 / 2+)}\right) /\left(2 C_{10}\right)$ satisfies (7-2) by (3-8), we can use Result 17 and show that there exists a subinterval $J^{\prime}=\left[t_{1}^{\prime}, t_{2}^{\prime}\right]$ such that $\left|t_{2}^{\prime} / t_{1}^{\prime}\right| \sim E^{10+} \eta^{-(36+)}$ and

$$
\|u\|_{L_{t}^{\infty} L_{x}^{6}\left(\Gamma_{+}\left(J^{\prime}\right)\right)} \leq \frac{C_{9} \eta^{2+} E^{-(1 / 2+)} C_{10}}{2 C_{10}} \leq C_{9} \frac{\eta^{2+} E^{-(1 / 2+)}}{2} .
$$

Now we claim that $\|u\|_{L_{t}^{4} L_{x}^{12}\left(\Gamma_{+}\left(J^{\prime}\right)\right)} \leq \eta$. If not by (3-8) and Result 16 we have

$$
\|u\|_{L_{t}^{\infty} L_{x}^{6}\left(\Gamma_{+}\left(J^{\prime}\right)\right)} \geq C_{9} \eta^{2+} E^{-(1 / 2+)} .
$$

Contradiction with (7-5).

Proof of Result 16. We substitute $J^{\prime}$ for $\Gamma_{+}\left(J^{\prime}\right)$ in (5-1) to get

$$
\|f(u)\|_{L_{t}^{1} L_{x}^{2}\left(\Gamma_{+}\left(J^{\prime}\right)\right)} \lesssim E^{1 / 2} .
$$

By the Strichartz estimates (1-20) on the truncated cone $\Gamma_{+}\left(J^{\prime}\right)$ we have

$$
\|u\|_{L_{t}^{2+\epsilon} L_{x}^{(6(2+\epsilon)) / \epsilon}\left(\Gamma_{+}\left(J^{\prime}\right)\right)} \lesssim E^{1 / 2}
$$

after following similar steps to prove (5-2). Therefore

$$
\eta=\|u\|_{L_{t}^{4} L_{x}^{12}\left(\Gamma_{+}(J)\right)} \lesssim\|u\|_{L_{t}^{2+\epsilon} L_{x}^{(6(2+\epsilon)) / \epsilon}\left(\Gamma_{+}\left(J^{\prime}\right)\right)}^{(2+\epsilon)}\|u\|_{L_{t}^{\infty} L_{x}^{6}\left(\Gamma_{+}\left(J^{\prime}\right)\right)}^{1-((2+\epsilon) / 4)} \lesssim E^{(2+\epsilon) / 8}\|u\|_{L_{t}^{\infty} L_{x}^{6}\left(\Gamma_{+}\left(J^{\prime}\right)\right)}^{1-((2+\epsilon) / 4)} .
$$

Therefore (7-1) holds.

Proof of Result 17. By (7-2) we have $E \eta^{-18} \geq 1$. Let $n$ be the largest integer such that $2 n \leq 4 E \eta^{-18}$. This implies that $n \geq E \eta^{-18}$. Let $A:=E \eta^{-18}$. Now we consider the interval $\left[t_{1}, A^{2 n} t_{1}\right] \subset J$. We write $\left[t_{1}, A^{2 n} t_{1}\right]=\left[t_{1}, A^{2} t_{1}\right] \cup \cdots \cup\left[A^{2(n-1)} t_{1}, A^{2 n} t_{1}\right]$. We have

$$
\sum_{i=1}^{n} e\left(A^{2 i} t_{1}\right)-e\left(A^{2(i-1)} t_{1}\right) \leq 2 E
$$

and by the pigeonhole principle there exists $i_{0} \in[1, n]$ such that

$$
e\left(A^{2 i_{0}} t_{1}\right)-e\left(A^{2\left(i_{0}-1\right)} t_{1}\right) \lesssim \eta^{18}
$$

Now we choose $a:=A^{2\left(i_{0}-1\right)} t_{1}$ and $b \in\left[A^{2 i_{0}-1} t_{1}, A^{2 i_{0}} t_{1}\right]$. Let $t_{1}^{\prime}:=A^{2\left(i_{0}-1\right)} t_{1}, t_{2}^{\prime}:=A^{2 i_{0}-1} t_{1}$ and $J^{\prime}:=\left[t_{1}^{\prime}, t_{2}^{\prime}\right]$. We apply (3-7) and (7-2) to get

$$
\|u\|_{L_{t}^{\infty} L_{x}^{6}\left(\Gamma_{+}\left(\left[t_{1}^{\prime}, t_{2}^{\prime}\right]\right)\right)} \lesssim\|F(u)\|_{L_{t}^{\infty} L_{x}^{1}\left(\Gamma_{+}\left(\left[t_{1}^{\prime}, t_{2}^{\prime}\right]\right)\right)} \lesssim\left(E^{-1} \eta^{18}\left(E+E^{1 / 3}\right)+\eta^{18}+\eta^{6}\right)^{1 / 6} \lesssim \eta .
$$




\section{Proof of Lemma 11}

We have after computation of the derivative of $e(t)$

$$
\partial_{t} e(t) \geq \int_{|x|=t} F(u) d S,
$$

and integrating with respect of time

$$
\int_{I} \int_{|x| \leq t} g(u) u^{6}\left(t^{\prime}, x^{\prime}\right) d S d t^{\prime} \lesssim E .
$$

By using the space and time translation invariance

$$
\int_{J} \int_{\left|x^{\prime}-x\right|=\left|t^{\prime}-t\right|} g(u) u^{6}\left(t^{\prime}, x^{\prime}\right) d S d t^{\prime} \lesssim E .
$$

Therefore (1-15), (1-22), (7-14) and the Hölder inequality give us

$$
\begin{aligned}
\left|-\int_{J^{\prime}} \frac{\sin \left(t-t^{\prime}\right) D}{D} g(u) u^{5} d t^{\prime}\right| & =\left|\frac{1}{4 \pi\left|t-t^{\prime}\right|} \int_{\left|x^{\prime}-x\right|=\left|t^{\prime}-t\right|} g^{5 / 6}(u) u^{5} g^{1 / 6}(u) d S d t^{\prime}\right| \\
& \lesssim \int_{J^{\prime}} \frac{1}{\left|t-t^{\prime}\right|}\left(\int_{\left|x^{\prime}-x\right|=\left|t^{\prime}-t\right|} u^{6} g(u) d S\right)^{5 / 6}\left(\int_{\left|x^{\prime}-x\right|=\left|t^{\prime}-t\right|} g(u) d S\right)^{1 / 6} d t^{\prime} \\
& \lesssim g^{1 / 6}(M) \int_{J^{\prime}} \frac{1}{\left|t-t^{\prime}\right|^{2 / 3}}\left(\int_{\left|x^{\prime}-x\right|=\left|t^{\prime}-t\right|} u^{6} g(u) d S\right)^{5 / 6} d t^{\prime} \\
& \lesssim g^{1 / 6}(M) E^{5 / 6}\left(\int_{J^{\prime}} \frac{1}{\left|t-t^{\prime}\right|^{4}}\right)^{1 / 6} \lesssim g^{1 / 6}(M) \frac{E^{5 / 6}}{\operatorname{dist}^{1 / 2}\left(t, J^{\prime}\right)}
\end{aligned}
$$

Notice that

$$
u(t)=u_{l, t_{i}}(t)-\int_{t_{i}}^{t} \frac{\sin \left(t-t^{\prime}\right) D}{D} u^{5}\left(t^{\prime}\right) g\left(u\left(t^{\prime}\right)\right) d t^{\prime},
$$

for $i=1,2$. We get (3-11) from (7-15) and (7-16).

\section{Acknowledgements}

The author thanks Terence Tao for suggesting him this problem.

\section{References}

[Bahouri and Gérard 1999] H. Bahouri and P. Gérard, "High frequency approximation of solutions to critical nonlinear wave equations", Amer. J. Math. 121:1 (1999), 131-175. MR 2000i:35123 Zbl 0919.35089

[Bourgain 1999] J. Bourgain, "Global wellposedness of defocusing critical nonlinear Schrödinger equation in the radial case", J. Amer. Math. Soc. 12:1 (1999), 145-171. MR 99e:35208

[Ginibre and Velo 1995] J. Ginibre and G. Velo, "Generalized Strichartz inequalities for the wave equation", J. Funct. Anal. 133:1 (1995), 50-68. MR 97a:46047 Zbl 0849.35064

[Grillakis 1990] M. G. Grillakis, "Regularity and asymptotic behaviour of the wave equation with a critical nonlinearity", Ann. of Math. (2) 132:3 (1990), 485-509. MR 92c:35080 Zbl 0736.35067 
[Grillakis 1992] M. G. Grillakis, "Regularity for the wave equation with a critical nonlinearity", Comm. Pure Appl. Math. 45:6 (1992), 749-774. MR 93e:35073 Zbl 0785.35065

[Kapitanski 1994] L. Kapitanski, "Global and unique weak solutions of nonlinear wave equations", Math. Res. Lett. 1:2 (1994), 211-223. MR 95f:35158 Zbl 0841.35067

[Keel and Tao 1998] M. Keel and T. Tao, "Endpoint Strichartz estimates", Amer. J. Math. 120:5 (1998), 955-980. MR 2000d: 35018 Zbl 0922.35028

[Lindblad and Sogge 1995] H. Lindblad and C. D. Sogge, "On existence and scattering with minimal regularity for semilinear wave equations”, J. Funct. Anal. 130:2 (1995), 357-426. MR 96i:35087 Zbl 0846.35085

[Rauch 1981] J. Rauch, "I: The $u^{5}$ Klein-Gordon equation; II: Anomalous singularities for semilinear wave equations", pp. 335-364 in Nonlinear partial differential equations and their applications (Paris, 1978/1979), vol. 1, edited by H. Brezis and J. L. Lions, Res. Notes in Math. 53, Pitman, Boston, MA, 1981. MR 83a:35066 Zbl 0473.35055

[Shatah and Struwe 1993] J. Shatah and M. Struwe, "Regularity results for nonlinear wave equations", Ann. of Math. (2) 138:3 (1993), 503-518. MR 95f:35164 Zbl 0836.35096

[Shatah and Struwe 1994] J. Shatah and M. Struwe, "Well-posedness in the energy space for semilinear wave equations with critical growth”, Internat. Math. Res. Notices 7 (1994), 303-309. MR 95e:35132 Zbl 0830.35086

[Shatah and Struwe 1998] J. Shatah and M. Struwe, Geometric wave equations, Courant Lecture Notes in Mathematics 2 , Courant Institute of Mathematical Sciences, New York, 1998. MR 2000i:35135 Zbl 0993.35001

[Sogge 1995] C. D. Sogge, Lectures on nonlinear wave equations, Monographs in Analysis 2, International Press, Boston, MA, 1995. MR 2000g:35153 Zbl 1089.35500

[Struwe 1988] M. Struwe, "Globally regular solutions to the $u^{5}$ Klein-Gordon equation", Ann. Scuola Norm. Sup. Pisa Cl. Sci.

(4) 15:3 (1988), 495-513. MR 90j:35142 Zbl 0728.35072

[Tao 2006] T. Tao, "Spacetime bounds for the energy-critical nonlinear wave equation in three spatial dimensions", Dyn. Partial Differ. Equ. 3:2 (2006), 93-110. MR 2007c:35116

[Tao 2007] T. Tao, "Global regularity for a logarithmically supercritical defocusing nonlinear wave equation for spherically symmetric data”, J. Hyperbolic Differ. Equ. 4:2 (2007), 259-265. MR 2009b:35294 Zbl 1124.35043

Received 4 Nov 2008. Revised 7 Jun 2009. Accepted 21 Jul 2009.

TRISTAN RoY: triroy@math.ucla.edu

Department of Mathematics, University of California, Los Angeles, CA 90095, United States 


\title{
Analysis \& PDE
}

pjm.math.berkeley.edu/apde

EDITORS

\author{
EDITOR-IN-CHIEF \\ Maciej Zworski \\ University of California \\ Berkeley, USA
}

BOARD OF EDITORS

\begin{tabular}{|c|c|c|c|}
\hline Michael Aizenman & $\begin{array}{l}\text { Princeton University, USA } \\
\text { aizenman@math.princeton.edu }\end{array}$ & Nicolas Burq & $\begin{array}{l}\text { Université Paris-Sud 11, France } \\
\text { nicolas.burq @math.u-psud.fr }\end{array}$ \\
\hline Luis A. Caffarelli & $\begin{array}{l}\text { University of Texas, USA } \\
\text { caffarel@math.utexas.edu }\end{array}$ & un-Yung Alice Chang & $\begin{array}{l}\text { Princeton University, USA } \\
\text { chang@math.princeton.edu }\end{array}$ \\
\hline Michael Christ & $\begin{array}{l}\text { University of California, Berkeley, USA } \\
\text { mchrist@math.berkeley.edu }\end{array}$ & Charles Fefferman & $\begin{array}{l}\text { Princeton University, USA } \\
\text { cf@math.princeton.edu }\end{array}$ \\
\hline Ursula Hamenstaedt & $\begin{array}{l}\text { Universität Bonn, Germany } \\
\text { ursula@math.uni-bonn.de }\end{array}$ & Nigel Higson & $\begin{array}{l}\text { Pennsylvania State Univesity, USA } \\
\text { higson@ math.psu.edu }\end{array}$ \\
\hline Vaughan Jones & $\begin{array}{l}\text { University of California, Berkeley, USA } \\
\text { vfr@math.berkeley.edu }\end{array}$ & Herbert Koch & $\begin{array}{l}\text { Universität Bonn, Germany } \\
\text { koch@math.uni-bonn.de }\end{array}$ \\
\hline Izabella Laba & $\begin{array}{l}\text { University of British Columbia, Canada } \\
\text { ilaba@math.ubc.ca }\end{array}$ & Gilles Lebeau & $\begin{array}{l}\text { Université de Nice Sophia Antipolis, France } \\
\text { lebeau@unice.fr }\end{array}$ \\
\hline László Lempert & $\begin{array}{l}\text { Purdue University, USA } \\
\text { lempert@math.purdue.edu }\end{array}$ & Richard B. Melrose & $\begin{array}{l}\text { Massachussets Institute of Technology, USA } \\
\text { rbm@math.mit.edu }\end{array}$ \\
\hline Frank Merle & $\begin{array}{l}\text { Université de Cergy-Pontoise, France } \\
\text { Frank.Merle@u-cergy.fr }\end{array}$ & William Minicozzi II & $\begin{array}{l}\text { Johns Hopkins University, USA } \\
\text { minicozz@ math.jhu.edu }\end{array}$ \\
\hline Werner Müller & $\begin{array}{l}\text { Universität Bonn, Germany } \\
\text { mueller@math.uni-bonn.de }\end{array}$ & Yuval Peres & $\begin{array}{l}\text { University of California, Berkeley, USA } \\
\text { peres@stat.berkeley.edu }\end{array}$ \\
\hline Gilles Pisier & $\begin{array}{l}\text { Texas A\&M University, and Paris } 6 \\
\text { pisier@math.tamu.edu }\end{array}$ & Tristan Rivière & $\begin{array}{l}\text { ETH, Switzerland } \\
\text { riviere@math.ethz.ch }\end{array}$ \\
\hline Igor Rodnianski & $\begin{array}{l}\text { Princeton University, USA } \\
\text { irod@ math.princeton.edu }\end{array}$ & Wilhelm Schlag & $\begin{array}{l}\text { University of Chicago, USA } \\
\text { schlag@math.uchicago.edu }\end{array}$ \\
\hline Sylvia Serfaty & $\begin{array}{l}\text { New York University, USA } \\
\text { serfaty@ cims.nyu.edu }\end{array}$ & Yum-Tong Siu & $\begin{array}{l}\text { Harvard University, USA } \\
\text { siu@math.harvard.edu }\end{array}$ \\
\hline Terence Tao & $\begin{array}{l}\text { University of California, Los Angeles, USA } \\
\text { tao@math.ucla.edu }\end{array}$ & A Michael E. Taylor & $\begin{array}{l}\text { Univ. of North Carolina, Chapel Hill, USA } \\
\text { met@math.unc.edu }\end{array}$ \\
\hline Gunther Uhlmann & $\begin{array}{l}\text { University of Washington, USA } \\
\text { gunther@math.washington.edu }\end{array}$ & András Vasy & $\begin{array}{l}\text { Stanford University, USA } \\
\text { andras@math.stanford.edu }\end{array}$ \\
\hline Virgil Voiculescu & $\begin{array}{l}\text { University of California, Berkeley, USA } \\
\text { dvv@math.berkeley.edu }\end{array}$ & Steven Zelditch & $\begin{array}{l}\text { Johns Hopkins University, USA } \\
\text { szelditch@math.jhu.edu }\end{array}$ \\
\hline
\end{tabular}

\section{PRODUCTION}

apde@mathscipub.org

Paulo Ney de Souza, Production Manager Sheila Newbery, Production Edito

Silvio Levy, Senior Production Editor

See inside back cover or pjm.math.berkeley.edu/apde for submission instructions.

The subscription price for 2009 is US \$120/year for the electronic version, and \$180/year for print and electronic. Subscriptions, requests for back issues from the last three years and changes of subscribers address should be sent to Mathematical Sciences Publishers, Department of Mathematics, University of California, Berkeley, CA 94720-3840, USA.

Analysis \& PDE, at Mathematical Sciences Publishers, Department of Mathematics, University of California, Berkeley, CA 94720-3840 is published continuously online. Periodical rate postage paid at Berkeley, CA 94704, and additional mailing offices.

APDE peer-review and production is managed by EditFLOW ${ }^{\mathrm{TM}}$ from Mathematical Sciences Publishers.

PUBLISHED BY

mathematical sciences publishers

http://www.mathscipub.org

A NON-PROFIT CORPORATION

Typeset in IATEX

Copyright (C2009 by Mathematical Sciences Publishers 


\section{ANALYSIS \& PDE}

\section{Volume $2 \quad$ No. $3 \quad 2009$}

Global existence of smooth solutions of a 3D log-log energy-supercritical wave equation

TRISTAN ROY

Periodic stochastic Korteweg-de Vries equation with additive space-time white noise

TADAHIRO OH

Stability for strongly coupled critical elliptic systems in a fully inhomogeneous medium

305

OLIVIER DRUET and EMMANUEL HEBEY

Global regularity for a logarithmically supercritical hyperdissipative Navier-Stokes equation 361

TERENCE TAO 